\title{
Polynomial invariants of links in the projective space
}

\author{
by
}

Maciej Mroczkowski (Uppsala)

\begin{abstract}
The Homflypt and Kauffman skein modules of the projective space are computed. Both are free and generated by some infinite set of links. This set may be chosen to be $\left\{L_{n}: n \in \mathbb{N} \cup\{0\}\right\}$, where $L_{n}$ is an arbitrary link consisting of $n$ projective lines for $n>0$, and $L_{0}$ is an affine unknot.
\end{abstract}

\section{INTRODUCTION}

The celebrated Jones polynomial [6] was generalized shortly after its discovery to the Homfly [3] and Kauffman [7] polynomials, for links in $\mathbb{R}^{3}$. The Jones polynomial was extended from the case of links in $\mathbb{R}^{3}$ to $\mathbb{R} P^{3}$ by Drobotukhina [2] and to all lens spaces by Hoste-Przytycki [5].

In this paper, the Homfly and Kauffman polynomials are extended to links in $\mathbb{R} P^{3}$. The technique we use is similar to the technique of Lickorish and Millett [8]. They used heavily the notion of descending diagram for links in $\mathbb{R}^{3}$. Here, we use the notion of descending diagram for links in $\mathbb{R} P^{3}$, introduced by the author [9].

I wish to thank Oleg Viro for his help.

1.1. Background: $\mathbb{R}^{3}$ case. The Homfly and Kauffman polynomials of framed links in $\mathbb{R}^{3}$ are defined by skein relations.

For Homfly polynomial, the skein relations are:

$$
\begin{aligned}
& \left.x^{-1} /\left(s-s^{-1}\right)\right)( \\
& \rho=\left(x v^{-1}\right) \uparrow
\end{aligned}
$$

In these relations, links are represented by their fragments which contain differences from other links under consideration. Moreover, the Homfly polynomial of a link is written simply as the link itself.

2000 Mathematics Subject Classification: Primary 57M27.

Key words and phrases: skein modules, projective space. 
Relations (HI) and (HII) together with the assumption that the polynomial is equal to 1 on the unknot $\bigcirc$ determine the polynomial for any framed oriented link in $\mathbb{R}^{3}$, and this polynomial is invariant under isotopy of such links.

Similarly, for Kauffman polynomial the skein relations are:

$$
\begin{aligned}
& \searrow+\lambda=z()(+\smile) \\
& \supset=a
\end{aligned}
$$

Relations (KI) and (KII) together with the assumption that the polynomial is equal to 1 on the unknot $\bigcirc$ determine the polynomial for any framed unoriented link in $\mathbb{R}^{3}$, and this polynomial is invariant under isotopy of such links.

1.2. Homflypt and Kauffman skein modules. Homfly and Kauffman polynomials can be extended to links in any oriented 3-manifold through the notion of skein modules, introduced independently by Przytycki [10] and Turaev [11].

For an oriented 3-manifold $M$, the Homflypt skein module of $M$ is the module over the ring $\mathbb{Z}\left[x^{ \pm 1}, s^{ \pm 1},\left(s-s^{-1}\right)^{-1}, v^{ \pm 1}\right]$, generated by the isotopy classes of the framed oriented links in $M$, with relations (HI) and (HII). The Kauffman skein module of $M$ is the module over $\mathbb{Z}\left[a^{ \pm 1}, z^{ \pm 1}\right]$, generated by the isotopy classes of the framed unoriented links in $M$, with relations (KI) and (KII).

There is another version of the Homflypt skein module of $M$ with unframed oriented links. However, there is essentially no difference between the two versions if $M$ has no non-separating 2-spheres: this follows from the fact that in such $M$ there are infinitely many non-isotopic framed knots that correspond to any unframed knot (announced in [4] and proved in [1]). As in our paper the case of $\mathbb{R} P^{3}$ is considered, it is sufficient to consider only the framed version.

Note that if $M$ is $\mathbb{R}^{3}$, the Homflypt and Kauffman skein modules are free cyclic modules generated by the unknot. The Homfly polynomial (resp. Kauffman polynomial) of a framed oriented link (resp. framed unoriented link) is obtained by expressing the link by means of the unknot: in the corresponding skein module the link equals the corresponding polynomial multiplied with the unknot.

1.3. Unknotting links in $\mathbb{R} P^{3}$. For $n>0$, the standard oriented unlink in $\mathbb{R} P^{3}$ with $n$ non-contractible components, denoted by $L_{n}$, is presented in Figure $1\left(\mathbb{R} P^{3}\right.$ is represented as a ball $D^{3}$ with antipodal points of the 
bounding sphere identified). $L_{0}$ is the unknot $\bigcirc$. The (line) framing for each $L_{n}$ is the blackboard framing (note that for projective lines this gives Möbius bands).

The standard unoriented unlink in $\mathbb{R} P^{3}$ with $n$ non-contractible components, again denoted by $L_{n}$, is the link of Figure 1 with the orientations being disregarded. $L_{0}$ is the unknot $\bigcirc$. The framing for each $L_{n}$ is again the blackboard framing.

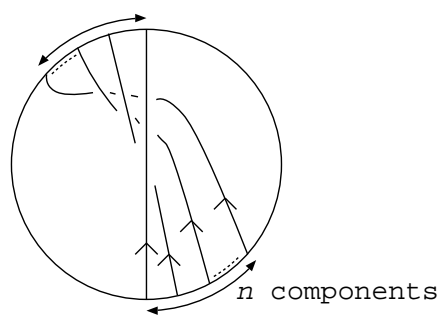

Fig. 1. Standard oriented unlink in $\mathbb{R} P^{3}$ with $n$ components

In [9] a notion of descending diagram for links in $\mathbb{R} P^{3}$ was introduced. It was shown that any diagram of an unoriented link can be transformed, by crossing changes alone, into a descending diagram (for a precise definition of diagrams of links in $\mathbb{R} P^{3}$, see Section 2.1 below). Such a descending diagram is a diagram of a link consisting of the disjoint union of $L_{n}$ (for some $n$ ) and some unknots $L_{0}$. Now, using induction on the number of crossings in a diagram, it follows that any unoriented projective link can be expressed as a linear combination of the $L_{n}$ 's in the Kauffman skein module of $\mathbb{R} P^{3}$.

A difficulty arises in the case of oriented links. Indeed, it was shown in [9] that it is not always possible to transform an arbitrary diagram into a diagram of the disjoint union of $L_{n}$ (for some $n$ ) and some unknots $L_{0}$, by crossing changes alone. However, it is possible to express any oriented projective link as a linear combination of some special links in the Homflypt skein module of $\mathbb{R} P^{3}$. These special links, as unoriented links, are equal to the unoriented $L_{n}$ 's. Now, using induction on the number of crossings in a diagram, one shows that any special link can be expressed as a linear combination of the oriented $L_{n}$ 's in the Homflypt skein module of $\mathbb{R} P^{3}$. Thus, any projective link can be expressed as a linear combination of the oriented $L_{n}$ 's in the Homflypt skein module of $\mathbb{R} P^{3}$. We have shown:

REMARK 1. The Homflypt skein module (resp. Kauffman skein module) of $\mathbb{R} P^{3}$ is generated by the standard oriented (resp. unoriented) unlinks $L_{n}$, $n \in \mathbb{N} \cup\{0\}$. 
In the rest of this paper, we establish that the two skein modules of $\mathbb{R} P^{3}$ are freely generated by the $L_{n}$ 's.

\subsection{Main theorems}

TheOREM 1. The Homflypt skein module of $\mathbb{R} P^{3}$ is freely generated by the standard unlinks $L_{n}, n \in \mathbb{N} \cup\{0\}$.

By Remark 1, this theorem is a consequence of the following:

THEOREM 2. To each framed oriented link $L \subset \mathbb{R} P^{3}$ one can associate a unique element $H(L) \in \mathbb{Z}\left[x^{ \pm 1}, s^{ \pm 1},\left(s-s^{-1}\right)^{-1}, v^{ \pm 1}, z\right]$ so that $H(L)$ depends only on the isotopy class of $L, H\left(L_{n}\right)=z^{n}$ for $n>0, H\left(L_{0}\right)=$ $\left(v^{-1}-v\right) /\left(s-s^{-1}\right)$, and relations (HI) and (HII) hold for $H$.

TheOREM 3. The Kauffman skein module of $\mathbb{R} P^{3}$ is freely generated by the standard unlinks $L_{n}, n \in \mathbb{N} \cup\{0\}$.

By Remark 1, this theorem is a consequence of the following:

THEOREM 4. To each framed unoriented link $L \subset \mathbb{R} P^{3}$ one can associate a unique element $K(L) \in \mathbb{Z}\left[a^{ \pm 1}, z^{ \pm 1}, y\right]$ so that $K(L)$ depends only on the isotopy class of $L, K\left(L_{n}\right)=y^{n}$ for $n>0, K\left(L_{0}\right)=\left(a+a^{-1}\right) z^{-1}-1$, and relations (KI) and (KII) hold for $K$.

The choices for $H\left(L_{0}\right)$ and $K\left(L_{0}\right)$ are convenient for constructions of $H$ and $K$. Also, with these choices, $H$ and $K$ are multiplicative under disjoint union. Note that in the case of projective links, the disjoint union is well defined on couples consisting of one affine link and one projective link.

In what follows, we will define the Homfly polynomial $H$ for framed oriented links in $\mathbb{R} P^{3}$ and the Kauffman polynomial $K$ for framed unoriented links in $\mathbb{R} P^{3}$. For technical reasons, the general notion of descending diagram (see Definition 3 in [9]) will not be used here, as it involves many choices. For our purpose, it is often easier to unknot only some part of the diagram of a link in order to obtain a simpler diagram. This approach will be used to define $H$ and $K$ by induction on the number of crossings in a diagram.

\section{DIAGRAMS AND BASIC DEFINITIONS}

2.1. Diagrams of links in the projective space and nets. A diagram of a link in $\mathbb{R} P^{3}$ is a disk with a collection of immersed arcs. An arc is a compact connected 1-manifold with or without boundary. The endpoints of arcs with boundary are on the boundary of the disk, divided into pairs of antipodal points and, with this restriction, the arcs are immersed generically. Each double point of the immersions or crossing of the diagram is endowed with information on over- and undercrossing. 
A net is the projective plane $\mathbb{R} P^{2}$ together with a distinguished (projective) line, called the line at infinity, and a collection of generically immersed circles endowed with over- and undercrossing information for each double point. We can map any diagram $D$ of a link to its net, obtained from $D$ by identifying the antipodal points of the boundary circle of $D$, with the line at infinity being the image of this boundary circle.

If $D$ is a diagram of a link $L$, and $L_{b}$ is a connected component of $L$, then the projection of $L$ onto $D$ maps $L_{b}$ onto a collection of arcs. Denote this collection by $b$. We will call $b$ a component of $D$ (though it may consist of several arcs). As $H_{1}\left(\mathbb{R} P^{3}\right)=\mathbb{Z}_{2}$ there are two types of connected components in a link: 0-homologous and non-0-homologous one. The corresponding components of a diagram of the link are said to be 0-homologous (their images in the net are contractible) and 1-homologous (their images in the net are not contractible).

If $D$ is a diagram of an oriented link $L$, then $D$ gets naturally oriented: each of its arcs is oriented. If $b$ is a component of $D$ coming from a component $L_{b}$ of $L$, then the orientation of $L$ gives rise to a cyclic ordering of the arcs of $b$ (when one travels on $L_{b}$ according to the orientation, one meets the arcs of $b$ in this order under the projection of $L$ onto $D$ ).

2.2. Arc distance, diagrams descending from $P$ to $Q$. Let $D$ be a diagram of an oriented link and $b$ a component of $D$. Let $P$ and $Q$ be two points in the interior of some arcs of $b$. Then the arc distance from $P$ to $Q$ is defined to be the number of times the line at infinity is crossed in the net of $D$ if one travels from the image of $P$ to the image of $Q$ in the net, according to the orientation of the image of $b$ in the net.

Suppose that $X$ is a crossing of $D$ such that at least one of its branches is in $b$. Then the first pass of $X$ from $P$ is, by definition, the branch of $X$ whose image in the net of $D$ is passed first if one travels from the image of $P$ in the net, according to the orientation of the image of $b$ in the net.

Suppose that $P$ and $Q$ are distinct. Then $D$ is said to be descending from $P$ to $Q$ if, for every crossing $X$ encountered when traveling from $P$ to $Q$ in the net according to the orientation, the first pass of $X$ from $P$ is an overpass (resp. underpass) whenever the arc distance from $P$ to this first pass is even (resp. odd). One says that $D$ is descending from $P$ to $P$ if it is descending from $P$ to $Q$, where $Q$ is a point on the same arc as $P$ and such that one can travel on this arc from $Q$ to $P$ according to the orientation without passing any crossing (i.e. $Q$ is just before $P$ ).

If $D$ is descending from $P$ to $Q$, then, traveling on the net from $P$ to $Q$, the encountered arcs are alternatively descending and ascending in the usual sense for diagrams of links in $\mathbb{R}^{3}$. 


\section{INDUCTIVE DEFINITION OF THE HOMFLY POLYNOMIAL $H$}

In this section the Homfly polynomial $H$ is constructed on diagrams with a given number of crossings, using the definition of $H$ on diagrams with strictly fewer crossings. We will assume that $H$ is constructed on diagrams with fewer than $n$ crossings and that it has some good properties (see Section 3.1 below). The construction of $H$ on diagrams with $n$ crossings requires endowing these diagrams with some extra structure: a basepoint or a couple of basepoints. The construction also depends, in some cases, on an ordering of a set of crossings of a diagram with $n$ crossings. In the next section we will show that $H$ does not depend on the choice of the extra structure or the choice of ordering, and that it has some good properties on diagrams with $n$ crossings or fewer.

A standard diagram of the standard oriented unlink $L_{n}$ is presented in Figure 1. Let $\mu=\left(v^{-1}-v\right) /\left(s-s^{-1}\right)$.

3.1. Inductive hypothesis $\operatorname{IH}(n-1)$. There is a function $H$ defined on the set of diagrams with at most $n-1$ crossings, taking values in $\mathbb{Z}\left[x^{ \pm 1}, s^{ \pm 1},\left(s-s^{-1}\right)^{-1}, v^{ \pm 1}, z\right]$, such that:

(1) $H$ is invariant under those Reidemeister moves that do not increase the number of crossings beyond $n-1$.

(2) $H$ satisfies relations (HI) and (HII).

(3) If $D$ is the standard diagram of the standard oriented unlink $L_{m}$, $m>0$, with at most $n-1$ crossings (i.e. $m(m-1) / 2 \leq n-1)$, then $H(D)=z^{m}$. Also, $H(\bigcirc)=\mu$.

3.2. Diagrams with no crossings. As the definition of $H$ uses induction on the number of crossings, $H$ is first defined for diagrams with 0 crossings.

Let $D$ be a diagram with 0 crossings. Let $p$ be the number of its 0 homologous components and $m$ the number of its 1-homologous components ( $m$ is 0 or 1 ). Then, by definition,

$$
H(D)=\mu^{p} z^{m}
$$

Note that $H$ satisfies $\mathrm{IH}(0)$.

3.3. Diagrams with $n \geq 1$ crossings. We assume that the inductive hypothesis $\mathrm{IH}(n-1)$ holds true.

The construction of $H$ for diagrams with $n$ crossings is divided into several cases treated in the subsequent subsections. In each case a diagram $D$ with $n$ crossings is endowed with some extra structure (a basepoint or a couple of basepoints). $D$ together with this structure is denoted by $\dot{D}$. 
A diagram $\alpha(\dot{D})$ is then defined: it is obtained from $\dot{D}$ by a series of crossing changes.

The diagram $\alpha(\dot{D})$ has the following property: if $X$ is one of the crossings of $\dot{D}$ that have to be switched to obtain $\alpha(\dot{D})$, and $\dot{D}^{\prime}$ is the diagram obtained from $\dot{D}$ by switching $X$, then $\alpha(\dot{D})=\alpha\left(\dot{D}^{\prime}\right)$.

In the following subsections $H$ is defined on $\alpha(\dot{D})$ for each case (see $\left(H_{3}\right)$ to $\left.\left(H_{6}\right)\right)$.

Suppose that $H$ is already defined on all $\alpha(\dot{D})$. For a based diagram $\dot{D}$, denote by $S(\dot{D})$ the set of crossings of $\dot{D}$ where $\dot{D}$ and $\alpha(\dot{D})$ differ. Let $k$ be the number of elements in $S(\dot{D})$ and $\omega$ a (linear) ordering of $S(\dot{D})$. Denote by $S(\dot{D}, \omega)$ the set $S(\dot{D})$ equipped with the ordering $\omega$.

We define $H(\dot{D}, \omega)$ by induction on $k$. The definition depends on $\omega$. If $k=0$ then $H$ is already defined. Otherwise let $\dot{D}^{\prime}$ be the based diagram obtained from $\dot{D}$ by switching the first crossing in $S(\dot{D}, \omega)$, and $D^{\prime \prime}$ be the diagram obtained by smoothing the same crossing. Let $\omega^{\prime}$ be the ordering of all crossings of $S\left(\dot{D}^{\prime}\right)$ induced by $\omega$. Note that there are $k-1$ elements in $S\left(\dot{D}^{\prime}\right) . H\left(\dot{D}^{\prime}, \omega^{\prime}\right)$ is defined by induction on $k$ and $H\left(D^{\prime \prime}\right)$ is defined by $\mathrm{IH}(n-1)$. Now $H(\dot{D}, \omega)$ is defined using relation (HI) for the first crossing in $S(\dot{D}, \omega)$ with the help of $H\left(\dot{D}^{\prime}, \omega^{\prime}\right)$ and $H\left(D^{\prime \prime}\right)$. Namely, if $\varepsilon$ is the sign of this first crossing, then, by definition,

$$
H(\dot{D}, \omega)=x^{2 \varepsilon} H\left(\dot{D}^{\prime}, \omega^{\prime}\right)+\varepsilon x^{\varepsilon}\left(s-s^{-1}\right) H\left(D^{\prime \prime}\right) .
$$

3.4. Simple diagrams. A diagram is said to be simple if it has at least one 1-homologous component and any of its crossings involves two different 1-homologous components. Thus, a simple diagram has no crossings involving 0-homologous components and no self-crossings of 1-homologous components.

A based simple diagram $\dot{D}$ (the dot indicates that the diagram is based) is a simple diagram $D$ equipped with a couple of basepoints. The basepoints are antipodal points that lie on the boundary circle of the diagram and are endpoints of some $\operatorname{arc}(\mathrm{s})$ of a 1-homologous component. They are indicated by black dots.

The antipodal basepoints are also called primary basepoints. They give rise to an ordering of 1-homologous components and a couple of antipodal basepoints, called secondary, on each of them (except the component that has the primary basepoints), in the following way: if one travels on the boundary of the disk from the primary basepoints in the counterclockwise direction, one encounters, for each 1-homologous component, a couple of endpoints belonging to it. For each such component, the couple encountered for the first time is by definition the couple of secondary basepoints. It is indicated by white dots. The 1-homologous components are ordered starting 
with the component with the primary basepoints, then the component whose secondary basepoints are encountered first and so on, until the component whose secondary basepoints are encountered last. An example of a based simple diagram with a couple of primary basepoints and two couples of secondary basepoints is shown in Figure 2.

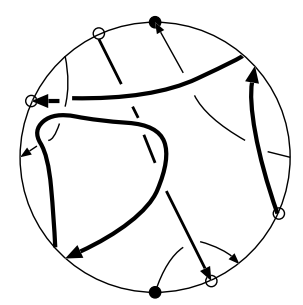

Fig. 2. Simple based diagram with 3 components

Denote by $\dot{D}_{k, l}$ the based diagram shown in Figure 3. It is called a standard based diagram. A based simple diagram $\dot{D}$ is said to be almost standard if it can be transformed into some $\dot{D}_{k, l}$ by removing all 0-homologous components and performing a (possibly empty) series of crossing changes.

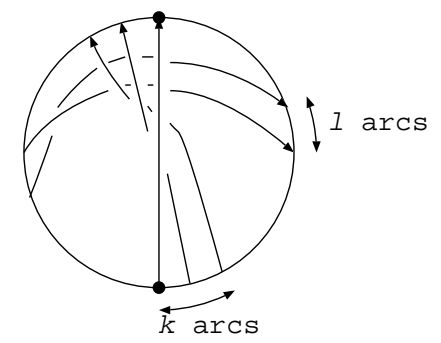

Fig. 3. Standard based diagram $\dot{D}_{k, l}$

For any almost standard diagram $\dot{D}$, let $\alpha(\dot{D})$ be the based diagram obtained from $\dot{D}$ by switching all the crossings with sign -1 . Thus $\alpha(\dot{D})$ has +1 sign at each crossing. Note that $\alpha(\dot{D})$ is a diagram of the disjoint union of $L_{m}$ (for some $m$ ) and some $L_{0}$ 's.

Let $p$ be the number of 0 -homologous components of $\dot{D}$ and $m$ the number of its 1-homologous components. Then, by definition,

$$
H(\alpha(\dot{D}))=\mu^{p} z^{m} .
$$

An oriented arc with two endpoints has an initial endpoint and a final one. Notice that in an oriented diagram, any couple of antipodal endpoints consists of one initial and one final endpoint.

Let $\dot{D}$ be a simple based diagram. Consider the endpoint in the couple of primary basepoints of $\dot{D}$ which is initial. Now travel on the boundary of 
the disk in the counterclockwise direction from this endpoint and consider each encountered endpoint in the couples of secondary basepoints. It can be either initial or final. One says that a component is good if the endpoint is initial, and bad if it is final. The component with the primary basepoints is always good. Note that $\dot{D}_{k, l}$ has $k$ good components followed by $l$ bad ones.

One says that an $\operatorname{arc} b$ follows an $\operatorname{arc} a$ if the endpoint of $b$ that is initial, is antipodal to an endpoint of $a$ (which has to be final). An arc is above (resp. below) a component if, at each crossing involving the arc and the component, the upper (resp. lower) branch belongs to the arc.

A simple based diagram $\dot{D}$ is said to be descending if, for any 1-homologous component $b$, the arc of $b$ that contains the basepoint of $b$ which is initial, is above the components coming after $b$ according to the order given by the primary basepoints; the arc that follows it is below the same components; and so on alternatingly, for all $\operatorname{arcs}$ of $b$.

For any simple based diagram $\dot{D}$ that is not almost standard, let $\alpha(\dot{D})$ be the based diagram obtained from $\dot{D}$ by crossing changes that make it descending.

Consider the crossings of $\dot{D}_{k, l}$ with sign -1 . Each of them is a crossing between the $i$ th good and $j$ th bad component so it may be indexed by $(i, j)$. Let $\omega_{k, l}$ be the lexicographical order of the set of all crossings with sign -1 , indexed in this way.

Let $\dot{D}$ be a simple based diagram that is not almost standard. Let $p$ be the number of 0-homologous components of $\dot{D}, k$ the number of its good components and $l$ the number of bad ones. Then, by definition,

$$
H(\alpha(\dot{D}))=\mu^{p} H\left(\dot{D}_{k, l}, \omega_{k, l}\right) .
$$

Note that in the case of an almost standard diagram $\dot{D}, \alpha(\dot{D})$ is not descending unless there are only good components in $\dot{D}$. In what follows, it is more convenient to have $\dot{D}_{k, l}$ descending rather than $\alpha\left(\dot{D}_{k, l}\right)$.

3.5. Non-simple diagrams. Another notion of based diagram, different from the one used for simple diagrams, is needed in order to construct $H$ for non-simple diagrams. Let $D$ be a non-simple diagram. A basepoint can be of two different kinds:

(1) A point on a 0-homologous component in the interior of some arc and distinct from any crossing if this component is involved in some crossings (see Figure 4(a)).

(2) A self-crossing of a 1-homologous component (see Figure 4(b)).

As before, a diagram $D$ equipped with a basepoint is denoted by $\dot{D}$. 


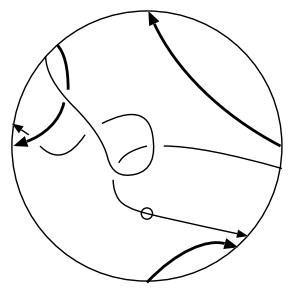

(a) 0-homologous

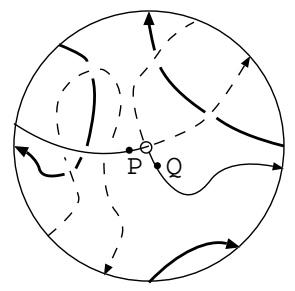

(b) 1-homologous

Fig. 4. Non-simple based diagrams

First, we consider $\dot{D}$ for which the basepoint is on a 0 -homologous component. $\dot{D}$ is said to be descending if it is descending from the basepoint to the basepoint (see Subsection 2.2). If $\dot{D}$ has a basepoint on a 0-homologous component, then $\alpha(\dot{D})$ is the based diagram obtained from $\dot{D}$ by the crossing changes that are necessary to make it descending.

Let $D^{\prime}$ be obtained from $\dot{D}$ by removing the component with the basepoint (if $D^{\prime}$ is empty then $H\left(D^{\prime}\right)$ is replaced by 1 in $\left(H_{5}\right)$ below). Let $w$ be the sum of all signs at all self-crossings of the component with basepoint in $\alpha(\dot{D})$. Then, by definition

$$
H(\alpha(\dot{D}))=\mu\left(x v^{-1}\right)^{w} H\left(D^{\prime}\right) .
$$

Finally, we define $H$ in the case when the basepoint of $\dot{D}$ is a self-crossing of a 1-homologous component.

Let $X$ be a self-crossing of a 1-homologous component, say $b$. A smoothing at $X$ according to any orientation of $b$ gives rise to two components: one 0 -homologous, the other 1 -homologous. The dashed part determined by $X$ is the part of $D$ corresponding to the 0 -homologous component.

Let $P$ and $Q$ be two points on $b$ such that, with respect to the orientation, $P$ is just before $X, Q$ is just after $X$, and $P$ and $Q$ are not in the dashed part determined by $X$ (see Figure 4(b)). Then $\dot{D}$ is said to be descending if it is descending from $P$ to $Q$ (i.e. the dashed part is descending). If $\dot{D}$ has a basepoint which is a self-crossing of a 1-homologous component, then $\alpha(\dot{D})$ is the based diagram obtained from $\dot{D}$ by the crossing changes which are necessary to make it descending.

Let $D^{\prime}$ be obtained from $\dot{D}$ by removing the dashed part determined by the basepoint. Let $w$ be the sum of the signs at all self-crossings of the dashed part determined by the basepoint of $\alpha(\dot{D})$, including the basepoint (which is a self-crossing). Then, by definition,

$$
H(\alpha(\dot{D}))=\left(x v^{-1}\right)^{w} H\left(D^{\prime}\right) .
$$




\section{INDEPENDENCE FROM CHOICES FOR $H$, INVARIANCE UNDER REIDEMEISTER MOVES}

In this section, we show that the definition of $H$ on diagrams with $n$ crossings does not depend on the choices involved (of basepoint and ordering of some crossings). We also prove that relations (HI) and (HII) are satisfied for diagrams with $n$ crossings and that $H$ is invariant under those Reidemeister moves which do not increase the number of crossings beyond $n$. Thus, assuming that $H$ satisfies the inductive hypothesis $\operatorname{IH}(n-1)$, it is proven that it satisfies $\operatorname{IH}(n)$.

\subsection{Relations (HI) and (HII)}

LEMMA 1 (independence from ordering). Let $\dot{D}$ be a based diagram with $n$ crossings. Let $\omega$ and $\omega^{\prime}$ be two orderings of the set of crossings of $\dot{D}$ that are different in $\dot{D}$ and $\alpha(\dot{D})$. Then $H(\dot{D}, \omega)=H\left(\dot{D}, \omega^{\prime}\right)$.

Proof. By induction on the number of crossing differences between $\dot{D}$ and $\alpha(\dot{D})$ it is sufficient to prove that $H$ does not change if one switches the first two crossings according to $\omega$, say $C_{1}$ and $C_{2}$ with respective signs $\varepsilon_{1}$ and $\varepsilon_{2}$.

Denote by $\sigma_{1} \dot{D}$ (resp. $\sigma_{2} \dot{D}$ ) the diagram obtained from $\dot{D}$ by switching $C_{1}$ (resp. $C_{2}$ ). Also, denote by $\eta_{1} \dot{D}$ (resp. $\eta_{2} \dot{D}$ ) the diagram obtained from $\dot{D}$ by smoothing at $C_{1}$ (resp. $C_{2}$ ). First consider the sequence in which $C_{1}$ is switched before $C_{2}$ :

$$
\begin{aligned}
H(\dot{D}, \omega)= & x^{2 \varepsilon_{1}} H\left(\sigma_{1} \dot{D}\right)+\varepsilon_{1} x^{\varepsilon_{1}}\left(s-s^{-1}\right) H\left(\eta_{1} \dot{D}\right) \\
= & x^{2 \varepsilon_{1}} x^{2 \varepsilon_{2}} H\left(\sigma_{2} \sigma_{1} \dot{D}\right)+x^{2 \varepsilon_{1}} \varepsilon_{2} x^{\varepsilon_{2}}\left(s-s^{-1}\right) H\left(\eta_{2} \sigma_{1} \dot{D}\right) \\
& +\varepsilon_{1} x^{\varepsilon_{1}}\left(s-s^{-1}\right) H\left(\eta_{1} \dot{D}\right) .
\end{aligned}
$$

And switching $C_{2}$ before $C_{1}$ yields

$$
\begin{aligned}
H\left(\dot{D}, \omega^{\prime}\right)= & x^{2 \varepsilon_{2}} x^{2 \varepsilon_{1}} H\left(\sigma_{1} \sigma_{2} \dot{D}\right)+x^{2 \varepsilon_{2}} \varepsilon_{1} x^{\varepsilon_{1}}\left(s-s^{-1}\right) H\left(\eta_{1} \sigma_{2} \dot{D}\right) \\
& +\varepsilon_{2} x^{\varepsilon_{2}}\left(s-s^{-1}\right) H\left(\eta_{2} \dot{D}\right) .
\end{aligned}
$$

The first terms are equal. By the inductive hypothesis,

$$
\begin{aligned}
& H\left(\eta_{1} \dot{D}\right)=x^{2 \varepsilon_{2}} H\left(\sigma_{2} \eta_{1} \dot{D}\right)+\varepsilon_{2} x^{\varepsilon_{2}}\left(s-s^{-1}\right) H\left(\eta_{2} \eta_{1} \dot{D}\right), \\
& H\left(\eta_{2} \dot{D}\right)=x^{2 \varepsilon_{1}} H\left(\sigma_{1} \eta_{2} \dot{D}\right)+\varepsilon_{1} x^{\varepsilon_{1}}\left(s-s^{-1}\right) H\left(\eta_{1} \eta_{2} \dot{D}\right) .
\end{aligned}
$$

Substituting these expressions above, one sees that $H(\dot{D}, \omega)=H\left(\dot{D}, \omega^{\prime}\right)$.

Lemma 2. Let $D$ be a diagram with at most $n-1$ crossings. Suppose that there is a self-crossing $X$ of a component a of $D$ such that the arc distance from the upper branch to the lower branch of $X$ is even and $D$ is descending from the upper branch to the lower branch of $X$. Let $a^{\prime}$ be the part of a that is covered if one travels in the net from the upper branch to the lower 
branch of $X$. Let $D^{\prime}$ be obtained from $D$ by erasing $a^{\prime}$. Let $w$ be the sum of the signs of the crossings at which both branches belong to $a^{\prime}$ (including $X$ ). Then $H(D)=\left(x v^{-1}\right)^{w} H\left(D^{\prime}\right)$.

Proof. If $a$ is 1-homologous then by $\operatorname{IH}(n-1)$ one can calculate $H(D)$ by letting a basepoint be the self-crossing $X$. The conclusion then follows from $\left(H_{6}\right)$.

Suppose now that $a$ is 0 -homologous. The lemma is proven by induction. Let $k$ be the number of crossings in $D$. For $k=1$ (there has to be at least one crossing in $D)$ the lemma is true by $\left(H_{5}\right)$. Suppose that the lemma is true for $k<l \leq n-1$ and that $D$ has $k=l$ crossings. The orientation of $D$ induces orientations on the branches of $X$. To compute $H(D)$, put a basepoint on the upper branch of $X$, just before the crossing. Then $D^{\prime}$, obtained from $D$ by removing $a^{\prime}$, inherits the basepoint from $D$.

Now, when computing $H(D)$ and $H\left(D^{\prime}\right)$ one can use relation (HI) only for the crossings that appear both in $D$ and $D^{\prime}$ since $a^{\prime}$ is already descending with respect to the basepoint in $D$. Let $Y$ be a crossing in $D$ and $D^{\prime}$ that needs to be changed in order to make the components with basepoints descending. Let $\sigma$ be the operation of switching $Y$, and $\eta$ the operation of smoothing $Y$ with respect to the orientation. For simplicity, assume that the sign of $Y$ is +1 . Then, by (HI),

$$
\begin{aligned}
x^{-1} H(D)-x H(\sigma D) & =\left(s-s^{-1}\right) H(\eta D), \\
x^{-1} H\left(D^{\prime}\right)-x H\left(\sigma D^{\prime}\right) & =\left(s-s^{-1}\right) H\left(\eta D^{\prime}\right) .
\end{aligned}
$$

By induction, as $\eta D$ has $k-1$ crossings, $H(\eta D)=\left(x v^{-1}\right)^{w} H\left(\eta D^{\prime}\right)$. Also $H(\sigma D)=\left(x v^{-1}\right)^{w} H\left(\sigma D^{\prime}\right)$, which is proven easily by induction on the number of crossings that need to be changed in order to make $D$ and $D^{\prime}$ with basepoints descending.

Thus $H(D)=\left(x v^{-1}\right)^{w} H\left(D^{\prime}\right)$.

Proposition 1 (Homfly relations). Relation (HII) holds for $H$ when the diagram on the left has $n$ crossings. Relation (HI) holds for $H$ when the two based diagrams on the left have $n$ crossings and the same basepoint(s).

Proof. For relation (HII), using the definition of $H$ and $\mathrm{IH}(n-1)$, the statement can be easily verified by considering separately two cases:

- the kink appearing in (HII) is either in a dashed part determined by the basepoint, or in the 0-homologous component to which the basepoint belongs,

- the kink is not as in the first case.

The proof for (HI) is more difficult. Suppose that $\dot{D}$ is a based diagram with $n$ crossings. Let $C$ be a crossing of $\dot{D}$. Let $\sigma \dot{D}$ be obtained from $\dot{D}$ by switching $C$, and $\eta \dot{D}$ be obtained from $\dot{D}$ by smoothing $C$. Without loss of 
generality one may suppose that the sign of $C$ is +1 (otherwise the roles of $\dot{D}$ and $\sigma \dot{D}$ are switched). We want to show that

$$
x^{-1} H(\dot{D})-x H(\sigma \dot{D})=\left(s-s^{-1}\right) H(\eta \dot{D}) .
$$

First, suppose that $\dot{D}$ is simple, or that $C$ is in the dashed part determined by the basepoint, or that $C$ is in the 0-homologous component containing the basepoint. In these cases, in the definition of $H(\dot{D})$ or $H(\sigma \dot{D})$ relation (HI) is used at the crossing $C$. By Lemma 1 it can be used at the beginning. Thus (HI) holds for $H$ in these cases.

Consider now the remaining possibility for the crossing $C$ : suppose that $C$ is such that none of its branches is in the 0-homologous component with basepoint of $\dot{D}$, or in the dashed part determined by the basepoint.

If $\dot{D}$ is descending, let $D^{\prime}$ be obtained from $\dot{D}$ by erasing the 0-homologous component on which the basepoint lies, or the dashed part determined by the basepoint. Note that $C$ can be naturally viewed as a crossing in $D^{\prime}$. As $D^{\prime}$ has at most $n-1$ crossings, (HI) holds by $\mathrm{IH}(n-1)$ for $H\left(D^{\prime}\right), H\left(\sigma D^{\prime}\right)$ and $H\left(\eta D^{\prime}\right)$.

But $H(\dot{D}), H(\sigma \dot{D})$ and $H(\eta \dot{D})$ are expressed respectively in terms of $H\left(D^{\prime}\right), H\left(\sigma D^{\prime}\right)$ and $H\left(\eta D^{\prime}\right)$ in the same way. This follows from the definition of $H$, except for $H(\eta \dot{D})$ when the basepoint is a self-crossing of a 1-homologous component and $C$ is in the same component. But in this case, Lemma 2 is used to express $H(\eta \dot{D})$ by means of $H\left(\eta D^{\prime}\right)$. Thus (HI) holds for $H(\dot{D}), H(\sigma \dot{D})$ and $H(\eta \dot{D})$.

Now, if $\dot{D}$ is not descending, then (HI) for $H(\dot{D}), H(\sigma \dot{D})$ and $H(\eta \dot{D})$ is proved by induction on the number of crossings that have to be changed in order to make it descending. At a crossing that has to be changed, relation (HI) allows one to express $H(\dot{D}), H(\sigma \dot{D})$ and $H(\eta \dot{D})$ by means of diagrams with fewer crossings to be changed, for which (HI) holds by induction, and diagrams with at most $n-1$ crossings for which (HI) holds by $\mathrm{IH}(n-1)$.

The following lemma is a consequence of relation (HI):

Lemma 3 (basepoints and crossing changes). Let $\dot{D}_{1}$ and $\dot{D}_{2}$ be two based diagrams with $n$ crossings that differ only in the position of the basepoint $(s)$. Let $\dot{D}_{1}^{\prime}$ be obtained from $\dot{D}_{1}$ by a crossing change, and $\dot{D}_{2}^{\prime}$ be obtained from $\dot{D}_{2}$ by the same crossing change. Suppose that $H\left(\dot{D}_{1}\right)=H\left(\dot{D}_{2}\right)$. Then $H\left(\dot{D}_{1}^{\prime}\right)=H\left(\dot{D}_{2}^{\prime}\right)$.

Proof. (HI) allows expressing $H\left(\dot{D}_{1}\right)$ in terms of $H\left(\dot{D}_{1}^{\prime}\right)$ and $H$ of a third diagram with $n-1$ crossings. Similarly, it allows expressing $H\left(\dot{D}_{2}\right)$ in terms of $H\left(\dot{D}_{2}^{\prime}\right)$ and $H$ of the same diagram with $n-1$ crossings. By induction, for the diagram with $n-1$ crossings, $H$ does not depend on the choice of basepoint(s). Thus, as $H\left(\dot{D}_{1}\right)=H\left(\dot{D}_{2}\right)$, also $H\left(\dot{D}_{1}^{\prime}\right)=H\left(\dot{D}_{2}^{\prime}\right)$. 


\subsection{Basepoints for 0 -homologous components}

Lemma 4 (moving the basepoint). Suppose that $P$ is a basepoint lying on a 0-homologous component of a based diagram $\dot{D}$ with $n$ crossings. Let $c$ be the arc on which $P$ lies. Then $H$ does not change if $P$ is moved on $c$.

Proof. Denote by $a$ the 0 -homologous component containing $P$. It is sufficient to prove that $H$ does not change when the basepoint passes through a crossing as in Figure 5.
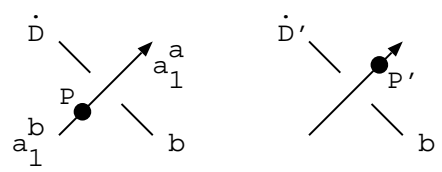

Fig. 5

Using Lemma 3, one may suppose that the diagram $\dot{D}$ on the left of Figure 5 is descending. Starting from $P$ and traveling on the net of $\dot{D}$ according to the orientation, denote the successive arcs encountered by $a_{1}, \ldots, a_{l}$. Furthermore denote the part of $a_{1}$ that comes after $P$ by $a_{1}^{a}$ and the remaining part by $a_{1}^{b}$. As $\dot{D}$ is descending, one has

$$
a_{2} \leq a_{4} \leq a_{6} \leq \cdots \leq a_{1}^{b} \leq \cdots \leq a_{5} \leq a_{3} \leq a_{1}^{a},
$$

where $a_{i} \leq a_{j}$ means that at each crossing involving $a_{i}$ and $a_{j}$ the branch in $a_{i}$ is under the branch in $a_{j}$, and $\leq$ is transitive.

Now $\dot{D}^{\prime}$ may be descending or not. If it is descending then $H(\dot{D})=H\left(\dot{D}^{\prime}\right)$ by $\left(H_{5}\right)$.

Suppose now that $\dot{D}^{\prime}$ is not descending. This is the case if and only if the branch $b$ (see Figure 5 ) is a part of $a_{1}^{a}, a_{1}^{b}$ or $a_{k}$ with $k$ odd. $\dot{D}^{\prime}$ becomes descending if one switches the crossing in Figure 5.

Let $\sigma \dot{D}^{\prime}$ be the based diagram obtained from $\dot{D}^{\prime}$ by this switching, and $\eta \dot{D}^{\prime}$ obtained from $\dot{D}^{\prime}$ by smoothing the same crossing. Note that in $\eta \dot{D}^{\prime}$, $a$ becomes a link with two 0-homologous components $a^{1}$ and $a^{2}$, where $a^{1}$ contains $P^{\prime}$ and $a_{2}$ contains $P\left(P\right.$ and $P^{\prime}$ can be naturally viewed in $\left.\eta \dot{D}^{\prime}\right)$. Notice that $\eta \dot{D}^{\prime}$ with basepoint $P^{\prime}$ is descending.

Denote by $D_{a}$ the diagram obtained from $\dot{D}^{\prime}$ by removing $a$. Notice that $D_{a}$ can be obtained from $\eta \dot{D}^{\prime}$ by removing $a^{1}$ and $a^{2}$. Denote by $D_{a^{1}}$ the diagram obtained from $\eta \dot{D}^{\prime}$ by removing $a^{1}$. Notice that $D_{a^{1}}$ with basepoint $P$ is descending. Let $\varepsilon= \pm 1$ be the sign of the crossing in Figure 5 .

For a 0-homologous component $c$ denote by $w(c)$ the sum of the signs at all crossings for which both branches are in $c$. Now: 


$$
\begin{aligned}
H(\dot{D}) & =\mu\left(x v^{-1}\right)^{w(a)} H\left(D_{a}\right), \\
H(\eta \dot{D}) & =\mu\left(x v^{-1}\right)^{w\left(a_{1}\right)} H\left(D_{a^{1}}\right)=\mu^{2}\left(x v^{-1}\right)^{w\left(a_{1}\right)+w\left(a_{2}\right)} H\left(D_{a}\right), \\
H\left(\dot{D}^{\prime}\right) & =x^{2 \varepsilon} H\left(\sigma \dot{D}^{\prime}\right)+\varepsilon x^{\varepsilon}\left(s-s^{-1}\right) H(\eta \dot{D}) \\
& =x^{2 \varepsilon} \mu\left(x v^{-1}\right)^{w(a)-2 \varepsilon} H\left(D_{a}\right)+\varepsilon x^{\varepsilon}\left(s-s^{-1}\right) \mu^{2}\left(x v^{-1}\right)^{w\left(a_{1}\right)+w\left(a_{2}\right)} H\left(D_{a}\right) .
\end{aligned}
$$

As $\mu=\left(v^{-1}-v\right) /\left(s-s^{-1}\right)$, we have

$$
H\left(\dot{D}^{\prime}\right)=\left(x^{w(a)} \mu v^{-w(a)+2 \varepsilon}+\varepsilon x^{\varepsilon} \mu\left(v^{-1}-v\right)\left(x v^{-1}\right)^{w\left(a_{1}\right)+w\left(a_{2}\right)}\right) H\left(D_{a}\right) .
$$

As $w\left(a_{1}\right)+w\left(a_{2}\right)=w(a)-\varepsilon$, we have

$$
H\left(\dot{D}^{\prime}\right)=\mu x^{w(a)} v^{-w(a)}\left(v^{2 \varepsilon}+\varepsilon v^{\varepsilon-1}-\varepsilon v^{\varepsilon+1}\right) H\left(D_{a}\right)=\mu\left(x v^{-1}\right)^{w(a)} H\left(D_{a}\right) .
$$

Since $\varepsilon=1$ or -1 , one checks easily that

$$
v^{2 \varepsilon}+\varepsilon v^{\varepsilon-1}-\varepsilon v^{\varepsilon+1}=1 \text {. }
$$

Thus $H(\dot{D})=H\left(\dot{D}^{\prime}\right)$.

4.3. Good Reidemeister moves. The Reidemeister moves presented in Figure 6 are called bad. They involve basepoint(s). For the bad moves, the usual notation is used, with one or more primes being added (e.g. $\Omega_{5}^{\prime \prime}$ ). All other moves are called good.

The moves $\Omega_{4}^{\prime}, \Omega_{5}^{\prime}$ and $\Omega_{5}^{\prime \prime}$ involve simple based diagrams. In an $\Omega_{4}^{\prime}$ move, for some component, the secondary basepoints are changed. An $\Omega_{5}^{\prime}$ move involves two couples of secondary basepoints and an $\Omega_{5}^{\prime \prime}$ move involves the primary and some secondary basepoints.

In this section, it is shown that $H$ does not change under good Reidemeister moves that do not involve diagrams with more than $n$ crossings. It will be proved in later sections that $H$ does not change under bad Reidemeister moves.

Using relation $(\mathrm{HI})$ and induction hypothesis $\mathrm{IH}(n-1)$ one easily gets the following:

REMARK 2 (crossing changes outside Reidemeister moves). Consider a Reidemeister move from a based diagram $\dot{D}_{1}$ to $\dot{D}_{2}$. If $H$ does not change under this move, then it does not change under the same move from $\dot{D}_{1}^{\prime}$ to $\dot{D}_{2}^{\prime}$, where $\dot{D}_{1}^{\prime}$ is obtained from $\dot{D}_{1}$ by switching some crossings not involved in the move, and $\dot{D}_{2}^{\prime}$ is obtained from $\dot{D}_{2}$ by switching the same crossings.

LEMMA 5 (crossing changes inside Reidemeister moves). Consider a Reidemeister move that is not an $\Omega_{4}$ move, from a based diagram $\dot{D}_{1}$ to $\dot{D}_{2}$. Let $b_{1}$ and $b_{2}$ be two branches involved in the move. Suppose that they are not the lowest and uppermost branch in an $\Omega_{3}$ move. If $H$ does not change under this move, then it does not change under any other move obtained from the first one by switching the crossing $(s)$ between $b_{1}$ and $b_{2}$. 


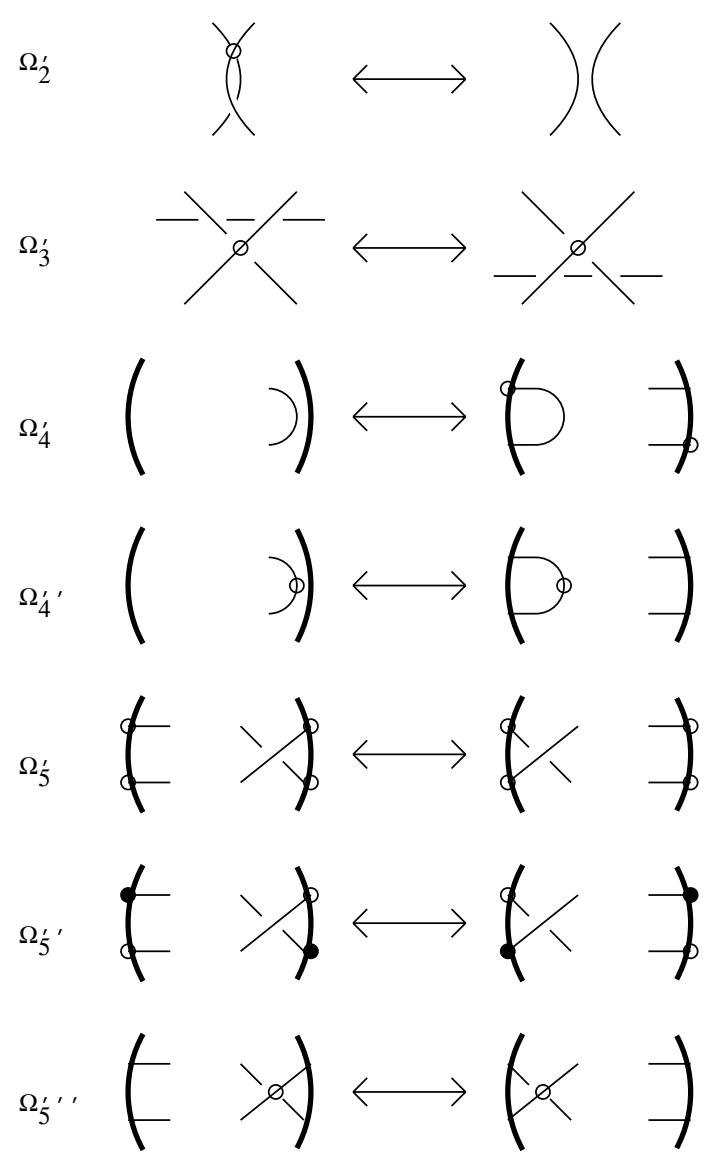

Fig. 6. Bad Reidemeister moves

Proof. For an $\Omega_{2}$ move, an easy calculation using (HI) and (HII) shows that $H$ is unchanged if one switches the two crossings that disappear under the move.

For an $\Omega_{3}$ move, let $\dot{D}_{1}^{\prime}$ (resp. $\dot{D}_{2}^{\prime}$ ) be obtained from $\dot{D}_{1}$ (resp. $\dot{D}_{2}$ ) by switching two adjacent branches (for example the lowest and the middle ones). Let $D_{1}^{\prime \prime}$ (resp. $D_{2}^{\prime \prime}$ ) be obtained from $\dot{D}_{1}$ (resp. $\dot{D}_{2}$ ) by smoothing the crossing that is switched to obtain $\dot{D}_{1}^{\prime}$ (resp. $\left.\dot{D}_{2}^{\prime}\right)$. By assumption $H\left(\dot{D}_{1}\right)=$ $H\left(\dot{D}_{2}\right)$. Now, $H\left(D_{1}^{\prime \prime}\right)=H\left(D_{2}^{\prime \prime}\right)$ because either the two diagrams are equal, or one can pass from one to the other by two $\Omega_{2}$ moves that do not increase the number of crossings beyond $n-1$ and one may use $\mathrm{IH}(n-1)$. From (HI) it follows that $H\left(\dot{D}_{1}^{\prime}\right)=H\left(\dot{D}_{2}^{\prime}\right)$.

The case of an $\Omega_{5}$ move is treated similarly to an $\Omega_{3}$ move. One uses two $\Omega_{4}$ moves between diagrams with $n-1$ crossings and $\operatorname{IH}(n-1)$ as well as (HI). 
Lemma 6 (invariance under good Reidemeister moves). $H$ does not change under good Reidemeister moves that involve diagrams with at most $n$ crossings.

Proof. By $\operatorname{IH}(n-1)$ it is sufficient to consider the case when at least one of the diagrams involved in the move has $n$ crossings. If the basepoint of the diagram with $n$ crossings lies on a 0 -homologous component, then by Lemma 4 it can be pushed out of the fragment of the diagram where the move takes place without changing $H$.

One may suppose that the diagram before the move has $n$ crossings and that it is descending, by Remark 2 and Lemma 5. It is easily checked that after the move the diagram is again descending except in a special case considered at the end of the proof. Now, from the definition of $H$ and $\mathrm{IH}(n-1)$ it follows easily that $H$ is unchanged under the move.

The special case is the following: the basepoint lies on a 0-homologous component and, after an $\Omega_{2}$ move, this component is not involved in any crossing. But again, in this case $H$ does not change by definition and $\mathrm{IH}(n-1)$.

4.4. Basepoints of simple diagrams. In this section it is shown that $H$ does not depend on the choice of antipodal basepoints for simple diagrams.

We say that an arc in a diagram $D$ separates a couple $P, Q$ of antipodal points lying on the boundary $S$ of the disk of $D$ if the endpoints of this arc are in different connected components of $S-(P \cup Q)$.

The following lemma is a reformulation of Lemma 1 of [9]:

LEMMA 7 (non-separating arcs for components with at least three arcs). Let $D$ be a simple diagram. Let $b$ be a 1-homologous component. Let $P, Q$ be a couple of antipodal points on the boundary circle of $D$, neither of them in $b$. Suppose that $b$ has at least three arcs. Then at least two of the arcs of $b$ do not separate $P, Q$.

LEMma 8 (non-separating arcs for components with at least five arcs). Let $D$ be a simple diagram and $b$ a 1-homologous component of D. Suppose that $b$ has at least five arcs. Consider two couples of antipodal endpoints of some arcs of $b$ such that moving from one couple to another in the counterclockwise direction, no other endpoints of $b$ are encountered. Then there is at least one arc with no endpoint in these two couples and which does not separate any of the two couples.

Proof. In Figure 7 four cases are presented. $P^{1}, Q^{1}$ and $P^{2}, Q^{2}$ are couples of antipodal endpoints. Suppose that there are no endpoints of $b$ between $P^{1}$ and $P^{2}$ (thus no endpoints between $Q^{1}$ and $Q^{2}$ ). 

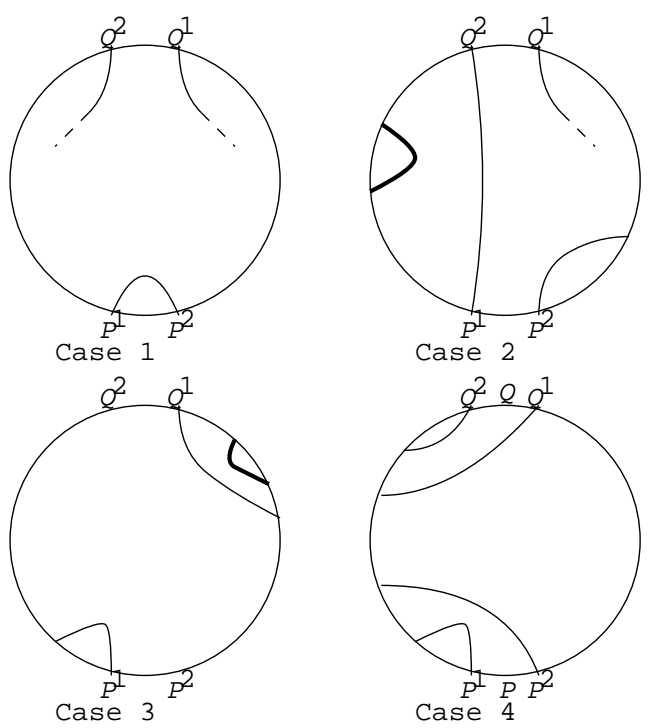

Fig. 7

CASE 1: $P^{1}$ and $P^{2}$ are endpoints of the same arc. The $\Omega_{4}$ move which deletes this arc, gives rise to a simple diagram in which $b$ has at least three arcs. This move changes only the three arcs shown in Figure 7, Case 1. By Lemma 7, at least two arcs of $b$ do not separate the antipodal endpoints $P^{1}$ and $Q^{1}$ (and also $P^{2}$ and $Q^{2}$ ). One of these may coincide with the arc obtained from the three arcs appearing in Case 1. But there is an extra arc not separating the couples of endpoints.

CAsE 2: $P^{1}$ and $Q^{2}$ are joined by an arc. Then the required arc obviously exists (the thick one).

CAsE 3: The arcs starting at $P^{1}$ and $Q^{1}$ do not separate the couple $P^{2}, Q^{2}$. Then one again finds a suitable arc (the thick one).

CASE 4: The only remaininig possibility is that some arcs with endpoints $P^{1}$ or $Q^{1}$ separate the couple $P^{2}, Q^{2}$ or vice versa. Suppose, for instance, that the arc which has $P^{2}$ as endpoint separates the couple $P^{1}, Q^{1}$. Then the arc with endpoint $P^{1}$ cannot separate $P^{2}, Q^{2}$ (the diagram is simple). Since we may assume we are not in the situation of Case 3, the arc with endpoint $Q^{1}$ has to separate $P^{2}, Q^{2}$. And this finally means that the arc with endpoint $Q^{2}$ does not separate $P^{1}, Q^{1}$.

Denote by $P, Q$ a couple of antipodal points on the boundary circle of the diagram that are not endpoints such that $P$ is between $P^{1}$ and $P^{2}$ and $Q$ is between $Q^{1}$ and $Q^{2}$.

Now we have two arcs which do not separate the couple $P, Q$ (namely, the arcs with endpoints $P^{1}$ and $Q^{2}$ ) and two arcs separating this couple 
(the arcs with endpoints $P^{2}$ and $Q^{1}$ ). Note that the two arcs that do not separate $P, Q$ are on the left side of the diagram.

To find a suitable arc in this case, let us prove first that the number of arcs of $b$ not separating $P, Q$ on the left side is the same as on the right side. This can be checked by induction on the number of arcs of $b$ : if $b$ has a unique arc this number is 0 on both sides. Otherwise, if one uses an $\Omega_{4}$ move to remove an arc of $b$ that does not separate $P, Q$ (which exists by Lemma 7), the number of arcs not separating this couple on the left and on the right decreases by one, or remains the same.

Thus there are at least two arcs of $b$ on the right side of the diagram that do not separate $P, Q$ and thus do not separate $P_{1}, Q_{1}$ and $P_{2}, Q_{2}$ either.

A subarc is a compact connected submanifold of an arc.

Let $a$ be a subarc going from a crossing to itself and having no other self-crossings. Let $P$ be a point on the arc of which $a$ is a subarc, just outside $a$. Then $a$ is called a 1-gon if, in the net, it does not separate $P$ and the line at infinity.

Let $\left(a_{1}, a_{2}\right)$ be a couple of subarcs both going from one crossing to another, having no extra crossings between them, and neither of them having self-crossings. Let $P_{1}$ be a point on an arc of which $a_{1}$ or $a_{2}$ is a subarc, just outside $a_{1}$ and $a_{2}$, and close to the first crossing between $a_{1}$ and $a_{2}$. Let $P_{2}$ be a point with the same properties as $P_{1}$ except that it is close to the second crossing between $a_{1}$ and $a_{2}$. Then $\left(a_{1}, a_{2}\right)$ is called a 2-gon if, in the net, it does not separate $P_{1}$ and the line at infinity, and it does not separate $P_{2}$ and the line at infinity.

1-gons and 2-gons are presented in Figure 8.
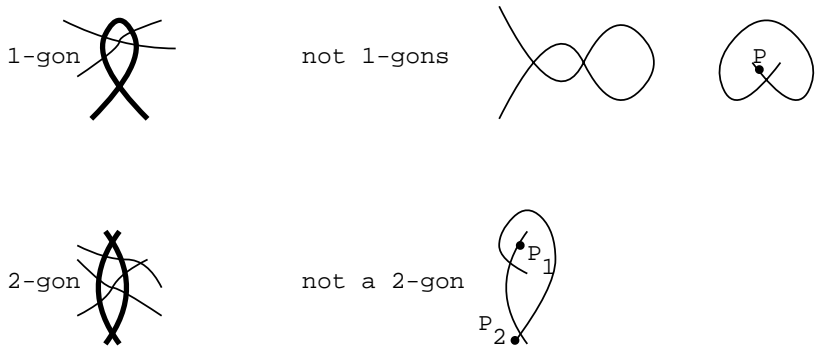

Fig. 8

By definition, a subarc $a$ is below a subarc $b$ if at each crossing involving $a$ and $b$ the branch in $a$ is under the branch in $b$.

Lemma 9 (moving subarcs). Let $D$ be a (possibly based) diagram with at most $n$ crossings. Let $B$ be a disk in $D$ such that there is no basepoint in $B$, and $B$ does not intersect the boundary circle of the diagram. Suppose 
that $B \cap D$ is the union of a subarc a strictly included in $\partial B$ and some other subarcs $a_{1}, \ldots, a_{l}$ properly embedded in $B$. Suppose that $a_{i}$ is below $a_{j}$ for any $i<j$ and there is $k$ such that $a$ is above $a_{i}$ for $i \leq k$ and below $a_{i}$ for $i>k$. Suppose that each $a_{i}$ crosses $a$ in one point and no pair of $a_{i}$ 's has more than one common crossing. Let $b$ be the closure of $\partial B-a$ and let $D^{\prime}$ be the result of substituting $b$ for $a$ in $D$, where $b$ is above $a_{i}$ if $a$ is, and below otherwise. Then $H(D)=H\left(D^{\prime}\right)$.

Proof. It is easily seen that it is possible to transform $D$ into $D^{\prime}$ by a series of good $\Omega_{3}$ moves.

Lemma 10 (removing 2-gons). Suppose that $B$ and $D$ are as in Lemma 9 except that one subarc $a_{i}$ crosses a in two points, and each $a_{j}, j \neq i$, crosses $a_{i}$ in one point. If, as before, $D^{\prime}$ is the result of substituting $b$ for a in $D$, then $H(D)=H\left(D^{\prime}\right)$.

Proof. Applying Lemma 9, move all crossings in the interior of the 2-gon formed by $a$ and $a_{i}$ out of this 2-gon. Transform the resulting diagram into $D^{\prime}$ by a series of good $\Omega_{3}$ moves and one good $\Omega_{2}$ move at the end.

Lemma 11 (removing 1-gons). Let $D$ be a (possibly based) diagram with at most $n$ crossings. Let a be a 1-gon bounding a disk B. Suppose that there are no 1-gons and no basepoint in $B$ (except possibly the self-crossing of a which can be a basepoint). Suppose that $B \cap D$ is the union of a and some other subarcs $a_{1}, \ldots, a_{l}$ properly embedded in $B$. Suppose that $a_{i}$ is below $a_{j}$ for any $i<j$ and there is $k$ such that $a$ is above $a_{i}$ for $i \leq k$ and $a$ is below $a_{i}$ for $i>k$. Let $\varepsilon$ be the sign of the self-crossing of a and $D^{\prime}$ the diagram obtained from $D$ by removing the 1-gon a. Then $H(D)=\left(x v^{-1}\right)^{\varepsilon} H\left(D^{\prime}\right)$.

Proof. First, remove all 2-gons that are inside $B$ using Lemma 10, starting with the most nested ones. Then decrease the number of crossings inside $B$ using Lemma 10 for couples of subarcs in which one subarc is part of $a$. In this way, the number of crossings inside $B$ is reduced to 0. Finally, use relation (HII) which holds for diagrams with at most $n$ crossings (Proposition 1).

LEMma 12 (special case of invariance under $\Omega_{5}^{\prime}$ moves). Suppose that an $\Omega_{5}^{\prime}$ move is applied to a simple based diagram $\dot{D}$ with $n$ crossings. Suppose that each of the two 1-homologous components involved in this move consists of a unique arc. Let $\dot{D}^{\prime}$ be the diagram after the move. Then $H(\dot{D})=H\left(\dot{D}^{\prime}\right)$.

Proof. By Remark 2 and Lemma 5 one may suppose that $\dot{D}$ is descending. If the two components involved in the move have at least three common crossings, then, using Lemma 10, remove two crossings that are not involved in the move without changing $H$ and, using $\operatorname{IH}(n-1)$, get $H(\dot{D})=H\left(\dot{D}^{\prime}\right)$. Otherwise, if the two components have a unique common crossing, then $\dot{D}^{\prime}$ 
is also descending, and both $H(\dot{D})$ and $H\left(\dot{D}^{\prime}\right)$ are equal to the same $H\left(\dot{D}_{k, l}\right)$ for some $k$ and $l$.

In the figures that follow, a sign of equality between two diagrams means that $H$ is the same for both. For simplicity, until the end of this subsection we will assume that the simple diagrams under consideration have no 0homologous components. This is possible as, by definition of $H$, each such component contributes to $\mu$ in the case of simple diagrams.

Proposition 2 (special case of independence from basepoints for simple diagrams). Let $\dot{D}_{1}$ and $\dot{D}_{2}$ be two simple based diagrams with $n$ crossings. Suppose that all 1-homologous components in $\dot{D}_{1}$ and $\dot{D}_{2}$ consist of unique arcs. Suppose that $\dot{D}_{1}$ and $\dot{D}_{2}$ differ only in the position of the basepoints. Then $H\left(\dot{D}_{1}\right)=H\left(\dot{D}_{2}\right)$.

Proof. It is sufficient to prove that $H\left(\dot{D}_{1}\right)=H\left(\dot{D}_{2}\right)$ if the basepoints of $\dot{D}_{2}$ are next to the basepoints of $\dot{D}_{1}$ in the counterclockwise direction.

By Lemma 3, one may suppose that $\dot{D}_{1}$ is descending. If there is a couple of components which have at least three crossings between them, then $H\left(\dot{D}_{1}\right)=H\left(\dot{D}_{2}\right)$ : indeed, one may reduce the number of crossings in $\dot{D}_{1}$ and $\dot{D}_{2}$ using Lemma 10 (because $\dot{D}_{1}$ is descending), and then use $\mathrm{IH}(n-1)$. Suppose now that any two components have a unique common crossing.

In changing the position of a couple of antipodal basepoints from $\dot{D}_{1}$ to $\dot{D}_{2}$, the following two cases can occur:

CASE 1: Traveling from the basepoint of $\dot{D}_{1}$ that is initial, in the counterclockwise direction, the first secondary basepoint encountered is also initial (see Figure 9).

One may suppose that $\dot{D}_{1}$ is in fact some $\dot{D}_{k, l}$. Indeed, if not, apply several times Lemma 9 and $\Omega_{5}^{\prime}$ moves to components with secondary basepoints (Lemma 12). Then, using Lemma 3, one may suppose that $\dot{D}_{1}$ is equal to $\alpha\left(\dot{D}_{k, l}\right)$, i.e. it is almost standard and the sign at every crossing is equal to +1 .

By $\left(H_{3}\right), H\left(\dot{D}_{1}\right)=z^{k+l}$. As shown in Figure $9, H\left(\dot{D}_{1}\right)=H\left(\dot{D}_{2}\right)$.

For Case 2, we need to have invariance of $H$ under some special $\Omega_{5}^{\prime \prime}$ move. Suppose that $\dot{D}$ is a simple based diagram with $n$ crossings such that each of its 1-homologous components consists of a unique arc. Suppose that one applies to $\dot{D}$ an $\Omega_{5}$ move involving primary and secondary basepoints ( $\Omega_{5}^{\prime \prime}$ move), and the vanishing triangle has two vertices that are both either initial basepoints or final endpoints. Suppose also that the two components involved in the move have a unique common crossing (it is the crossing that appears in the move). 

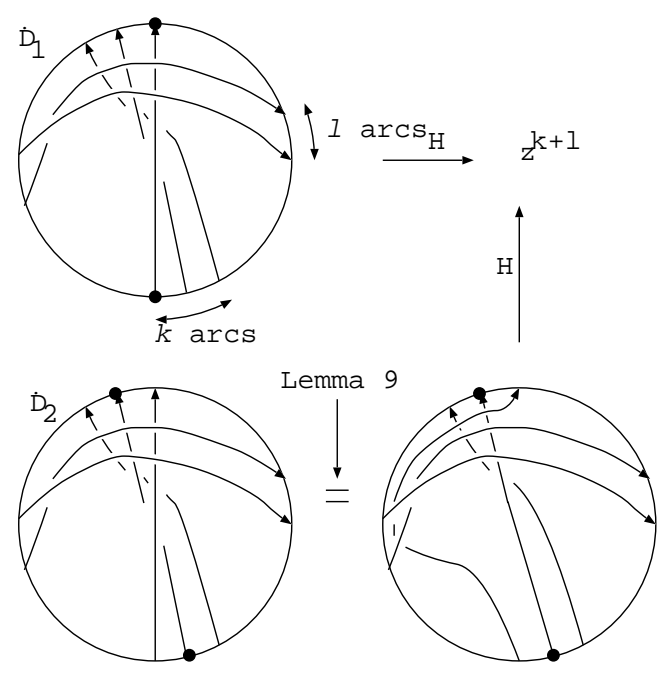

Fig. 9

Then $H$ does not change under such move. Indeed, using Remark 2 and Lemma 5 , suppose that $\dot{D}$ is descending. Before the move $H(\dot{D})=$ $H\left(\dot{D}_{k, l}\right)$ for some $k$ and $l$. After the move, change the basepoints in clockwise direction: as just proven above, $H$ does not change. The resulting based diagram is again descending and $H$ of this diagram is again equal to $H\left(\dot{D}_{k, l}\right)$.

CASE 2: Traveling from the basepoint of $\dot{D}_{1}$ that is initial, in the counterclockwise direction, the first secondary basepoint encountered is final (see Figure 10).

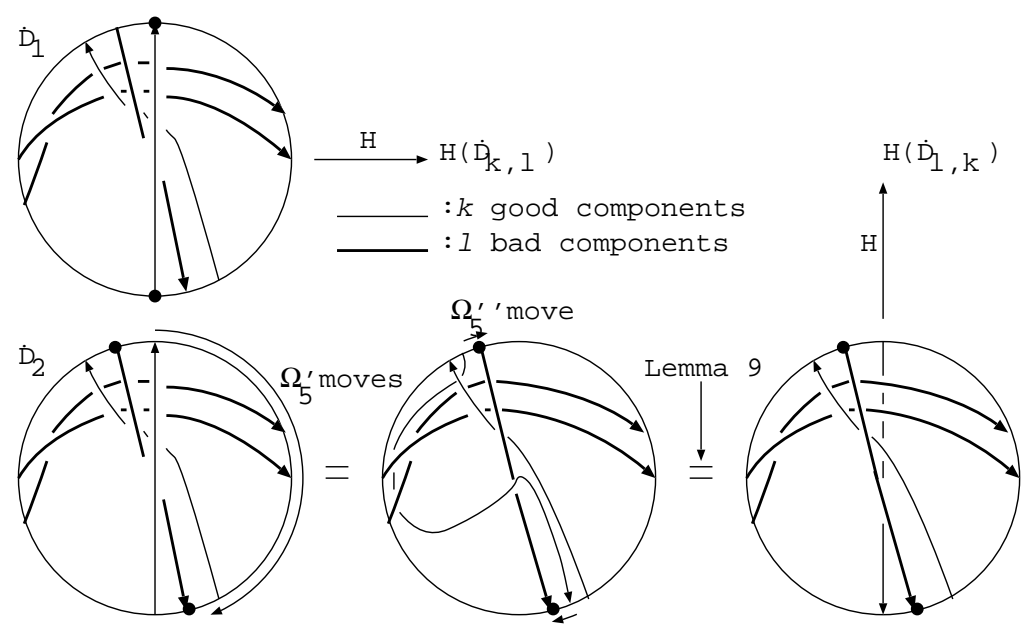

Fig. 10 


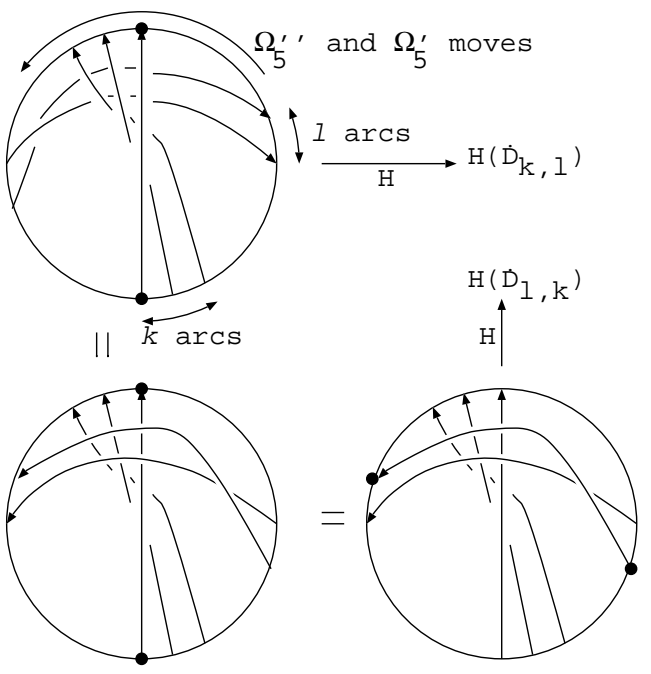

Fig. 11

Suppose that $\dot{D}_{1}$ is as on the top left of Figure 10. One may always reduce Case 2 to the situation presented in this figure, using Lemmas 3, 9 and 12 .

In this case, $H\left(\dot{D}_{1}\right)=H\left(\dot{D}_{k, l}\right)$, whereas $H\left(\dot{D}_{2}\right)=H\left(\dot{D}_{l, k}\right)$ (see Figure 10 where an $\Omega_{5}^{\prime \prime}$ move that does not change $H$ is applied). But, as shown in Figure $11, H\left(\dot{D}_{k, l}\right)=H\left(\dot{D}_{l, k}\right)$. In this figure, $\Omega_{5}^{\prime \prime}$ moves that do not change $H$ are applied. Also, Case 1 is used to change the position of primary basepoints without changing $H$.

Lemma 13 (special case of invariance under $\Omega_{5}^{\prime \prime}$ moves). Suppose that $\dot{D}$ is a simple based diagram with $n$ crossings, and all 1-homologous components of $\dot{D}$ consist of unique arcs. Let $\dot{D}^{\prime}$ be obtained from $\dot{D}$ by an $\Omega_{5}^{\prime \prime}$ move. Then $H(\dot{D})=H\left(\dot{D}^{\prime}\right)$.

Proof. Using Remark 2 and Lemma 5, assume that $\dot{D}$ is descending.

First, suppose that the two components involved in the move have at least three common crossings. Then $H(\dot{D})=H\left(\dot{D}^{\prime}\right)$, because one can remove two crossings by Lemma 10 and use $\operatorname{IH}(n-1)$. We may therefore assume that the two components involved have a unique common crossing.

If, in applying $\Omega_{5}^{\prime \prime}$, the vanishing triangle has two vertices that are both initial basepoints, or both final basepoints, then $H(\dot{D})=H\left(\dot{D}^{\prime}\right)$ as already seen in the proof of Proposition 2.

Suppose now that the vanishing triangle has one vertex that is an initial basepoint and another one that is a final basepoint. As $\dot{D}$ is descending, $H(\dot{D})=H\left(\dot{D}_{k, l}\right)$ for some $k$ and $l$. By moving the basepoints of $\dot{D}^{\prime}$ in the clockwise direction, one gets a descending diagram with $l$ good and $k$ 
bad components. Thus $H\left(\dot{D}^{\prime}\right)=H\left(\dot{D}_{l, k}\right)$. But it was seen in the proof of Proposition 2 that $H\left(\dot{D}_{k, l}\right)=H\left(\dot{D}_{l, k}\right)$.

Proposition 3 (independence from basepoints for simple diagrams). Let $\dot{D}_{1}$ and $\dot{D}_{2}$ be two simple based diagrams with $n$ crossings. Suppose that $\dot{D}_{1}$ and $\dot{D}_{2}$ differ only in the position of the basepoints. Then $H\left(\dot{D}_{1}\right)=$ $H\left(\dot{D}_{2}\right)$.

Proof. It is sufficient to prove that $H\left(\dot{D}_{1}\right)=H\left(\dot{D}_{2}\right)$ if the basepoints of $\dot{D}_{2}$ (say $\left.\left(P_{2}, Q_{2}\right)\right)$ are next to the basepoints of $\dot{D}_{1}$ (say $\left.\left(P_{1}, Q_{1}\right)\right)$ in the counterclockwise direction. By Lemma 3 , we may assume that $\dot{D}_{1}$ is descending. Let $a$ be the component to which $\left(P_{1}, Q_{1}\right)$ belongs. Then $\left(P_{2}, Q_{2}\right)$ may belong to the same component $a$ or to a different one, say $b$.

CASE 1: $\left(P_{2}, Q_{2}\right)$ belongs to $a$. Note that the secondary basepoints of $\dot{D}_{2}$ coincide with the secondary basepoints of $\dot{D}_{1}$. Thus, the ordering of components of $\dot{D}_{1}$ arising from the primary basepoints is the same for $\dot{D}_{2}$. Consider, for this ordering, the last component (if any) that has more than one arc. In Figure 12, this component is dashed.
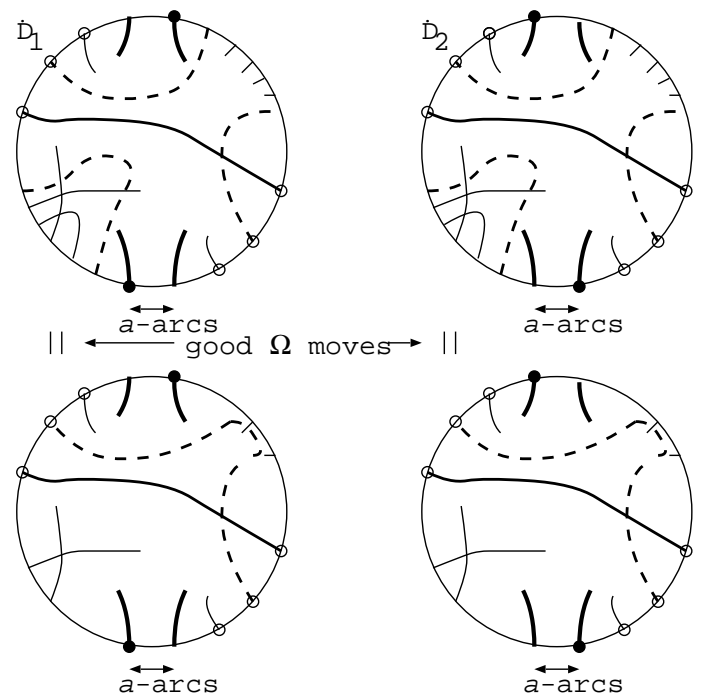

Fig. 12

One can reduce the number of arcs of this component without changing $H$ for both $\dot{D}_{1}$ and $\dot{D}_{2}$. By Lemma 7 , the component has an arc, say $c$, that does not separate its secondary couple of endpoints. Consider an arc that is most nested in $c$. This arc cannot have an endpoint that is a secondary basepoint. Using Lemmas 9,10 and good $\Omega_{5}$ and $\Omega_{4}$ moves (good in $\dot{D}_{1}$ and in $\dot{D}_{2}$ ), we remove this arc without changing $H$. The diagram obtained in 
this way from $\dot{D}_{1}$ is still descending. In the same manner, all arcs nested in $c$ are removed. Finally, $c$ is removed.

In this way, without changing $H, \dot{D}_{1}$ is transformed into a descending based diagram (still denoted by $\dot{D}_{1}$ ) in which all components, except $a$, have unique arcs. $\dot{D}_{2}$ is transformed similarly. Now by Lemma 8 the number of arcs of $a$ is reduced to three by eliminating the arcs that do not separate the couples $\left(P_{1}, Q_{1}\right)$ and $\left(P_{2}, Q_{2}\right)$, and that have no endpoints among $P_{1}$, $Q_{1}, P_{2}, Q_{2}$. During the elimination, $H$ is unchanged for $\dot{D}_{1}$ and $\dot{D}_{2}$, and $\dot{D}_{1}$ stays descending. One arrives at the situation presented in Figure 13. In this figure the arcs of $a$ (the thickest ones) are marked with high if they are above everything else, or low if they are below everything else.

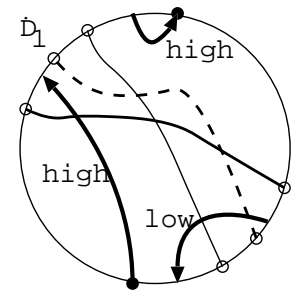

II

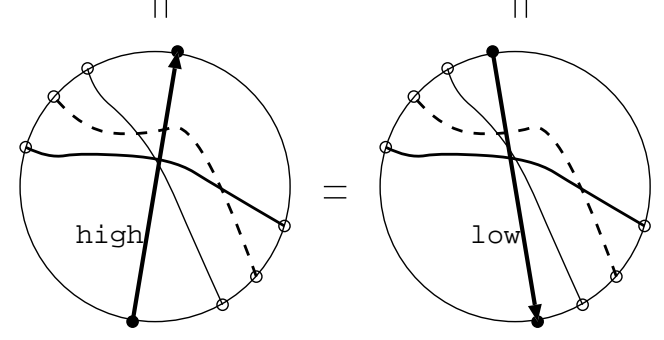

Fig. 13

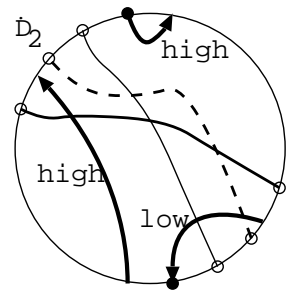

II (1)

At the bottom of the figure, the equality holds because one may turn around the component $a$ by moving some subarcs (Lemma 9) and by application of several $\Omega_{5}^{\prime \prime}$ moves in the situation where all components have unique arcs. It follows from Lemma 13 that $H$ does not change.

CASE 2: $\left(P_{2}, Q_{2}\right)$ belongs to a component $b$ different from $a$. A first possibility is that $a$ has a unique arc. In that case, as in Case 1 , one can reduce the number of arcs without changing $H$, and get diagrams for which all components have unique arcs. Then it follows from Proposition 2 that $H$ is unchanged.

Otherwise $a$ has several arcs. The method of Case 1 can be repeated for components that have secondary basepoints after the secondary basepoints of $a$ in $\dot{D}_{2}$. For each of these components the number of arcs is reduced to one. Also, the number of arcs of $a$ is reduced to three with the help of 
Lemma 8. Now, one has to compare the diagrams at the top of Figure 14. In this figure, the arcs of $a$ (thickest) are again marked with high or low. Note that in the bottom rightmost diagram of the figure, the unique arc of $a$ is divided into two parts: one high and the other low.
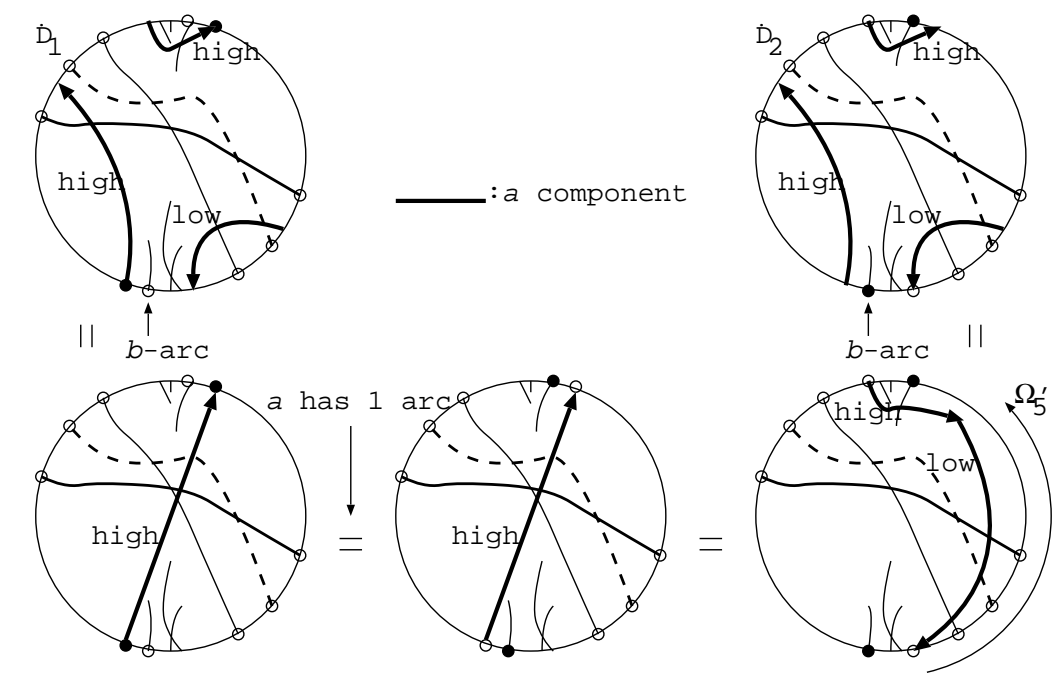

Fig. 14

In the bottom rightmost diagram of Figure 14, one applies several $\Omega_{5}^{\prime}$ moves involving $a$, which has a unique arc, and another component with a unique arc. By Lemma 12, $H$ does not change under such moves. In general, one also applies good $\Omega_{5}$ moves involving $a$ and some components that have secondary basepoints before the secondary basepoints of $a$ in $\dot{D}_{2}$. One may also have to eliminate some arcs of these components using Lemma 9 and good $\Omega_{5}$ and $\Omega_{4}$ moves.

Finally, $H$ is the same on the left and right side of Figure 14.

\section{5. $\Omega_{4}^{\prime}, \Omega_{5}^{\prime}$ and $\Omega_{5}^{\prime \prime}$ moves}

Proposition 4 (invariance under $\Omega_{4}^{\prime}, \Omega_{5}^{\prime}$ and $\Omega_{5}^{\prime \prime}$ moves). $H$ does not change under $\Omega_{4}^{\prime}$, $\Omega_{5}^{\prime}$ and $\Omega_{5}^{\prime \prime}$ moves, involving diagrams with $n$ crossings.

Proof. Recall that an $\Omega_{4}^{\prime}$ move is an $\Omega_{4}$ move under which the secondary basepoints of some component are changed. Consider the component involved in an $\Omega_{4}^{\prime}$ move and its secondary basepoints in the diagram with fewer arcs (i.e. the diagram before the move if we assume that $\Omega_{4}^{\prime}$ goes from a diagram with fewer arcs to a diagram with more arcs). By changing the position of the basepoints (this leaves $H$ unchanged according to Proposition 3) make these basepoints primary in both diagrams involved in the move. Then $\Omega_{4}^{\prime}$ is transformed into a good $\Omega_{4}$ move, so $H$ is unchanged. 
An $\Omega_{5}^{\prime}$ move involving two components which have unique arcs leaves $H$ unchanged according to Lemma 12. Now, if a component involved in an $\Omega_{5}^{\prime}$ move has at least three arcs then, by changing the position of the basepoints, the move can be transformed into a good $\Omega_{5}$ move. Thus any $\Omega_{5}^{\prime}$ move leaves $H$ unchanged.

If all components have unique arcs then an $\Omega_{5}^{\prime \prime}$ move leaves $H$ unchanged according to Lemma 13. Now if there is a component with at least three arcs, one can transform the $\Omega_{5}^{\prime \prime}$ move into an $\Omega_{5}$ or $\Omega_{5}^{\prime}$ move, by changing the position of the basepoints. It follows that any $\Omega_{5}^{\prime \prime}$ move leaves $H$ unchanged.

4.6. $\Omega_{2}^{\prime}$ and $\Omega_{3}^{\prime}$ moves. In this section, the invariance of $H$ under the moves $\Omega_{2}^{\prime}$ and $\Omega_{3}^{\prime}$ is established as a consequence of Proposition 5 below.

LEMma 14 (removing 1-gons, stronger version). Let $D$ be a diagram with at most $n$ crossings. Let a be a 1-gon bounding a disk B. Suppose that there is no basepoint in $B$ (except possibly the self-crossing of a which can be a basepoint), and $B \cap D$ is the union of $a$ and some other subarcs $a_{1}, \ldots, a_{l}$ properly immersed in $B$. Suppose that there is $k$ such that a is above $a_{i}$ for $i \leq k$, and below $a_{i}$ for $i>k$. Suppose also that if $i \leq k$ and $j>k$ then $a_{i}$ is below $a_{j}$. Let $\varepsilon$ be the sign of the self-crossing of a and $D^{\prime}$ the diagram obtained from $D$ by removing the 1-gon a. Then $H(D)=\left(x v^{-1}\right)^{\varepsilon} H\left(D^{\prime}\right)$.

Proof. The proof is by induction on the number of crossings in the interior of $B$, say $m$. If $m=0$ then one may apply Lemma 11 .

If there are no 1-gons in the interior of $B$, use relation (HI) for both $D$ and $D^{\prime}$ to order the subarcs $a_{1}, \ldots, a_{l}$ so that $a_{i}$ is below $a_{j}$ if $i<j$. Using (HI) gives rise to smoothings for which one applies induction on $m$. Now for the diagrams in which $a_{1}, \ldots, a_{l}$ are ordered, one applies Lemma 11.

If there are some 1-gons in the interior of $a$, consider one of them that is most nested (i.e. there are no 1-gons in its interior). It can be eliminated as in the preceding paragraph and one applies induction on $m$.

Lemma 15 (removing triangles). Suppose that in a diagram $D$ with at most $n-1$ crossings, one has the situation presented on the left of Figure 15. At the bottom of $D$ two subarcs a and $b$ (the thick ones), together with a part of the boundary circle of $D$, form a triangle. Inside the triangle, there are properly embedded subarcs $a_{1}, \ldots, a_{l}$, all intersecting $a$ and $b$ in a unique crossing. Suppose that no pair of $a_{i}$ 's has more than one crossing. Suppose that $a_{i}$ is below $a_{j}$ if $i<j$ and that there are $k$ and $k^{\prime}$ such that $a$ is above $a_{i}$ for $i \leq k$ and below $a_{i}$ for $i>k$, and $b$ is above $a_{i}$ for $i \leq k^{\prime}$ and below $a_{i}$ for $i>k^{\prime}$. Suppose that $a$ is below $b$ if $k \leq k^{\prime}$ and above $b$ otherwise. Then $H$ is the same for the diagrams on the left and on the right of Figure 15, where $a, b$ and the $a_{i}$ 's are above or below each other on the right in the same way as they are on the left. 

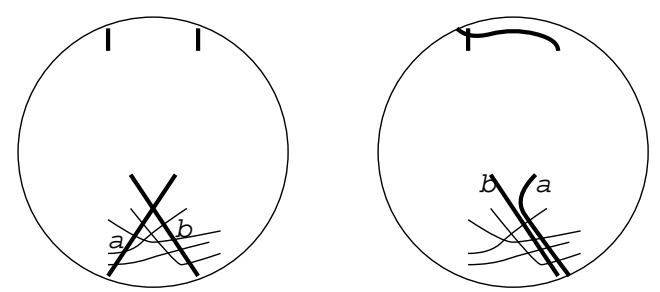

Fig. 15

Proof. The proof is similar to that of Lemma 9. The diagram on the left of Figure 15 is transformed into the diagram on the right by several $\Omega_{3}$ moves and one $\Omega_{5}$ move. This transformation is sketched in Figure 16.

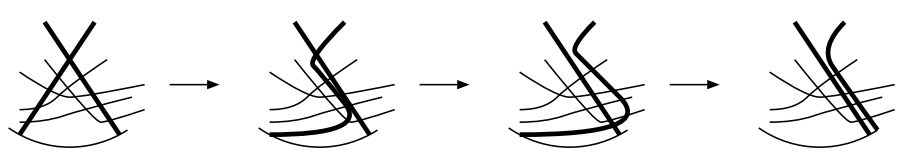

Fig. 16

Proposition 5 (shortening of diagrams). Let $D$ be a diagram with $k$ crossings. A part of $D$ is shown on the left of Figure 17. Suppose that the arc distance from $P$ to $Q$ is even and $D$ is descending from $P$ to $Q$. The part of $D$ from $P$ to $Q$ is dashed. $D^{\prime}$ is obtained from $D$ by removing this dashed part, and joining $P$ and $Q$ with a segment (a part of $D^{\prime}$ is shown on the right of Figure 17). Let $w$ be the sum of the signs at all crossings for which both branches are in the dashed part. If $k \leq n-1$ then $H(D)=\left(x v^{-1}\right)^{w} H\left(D^{\prime}\right)$.

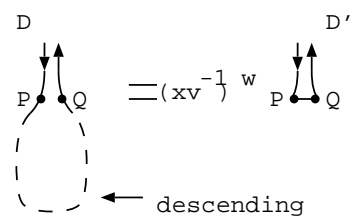

Fig. 17

Proof. The proof is by induction on $k$, the number of crossings in $D$. If $k=0$ then $H(D)=H\left(D^{\prime}\right)$ by definition of $H$.

Suppose the assertion is true for $k<l$, where $l \leq n-1$. Suppose now that $k=l$. We will use induction on the arc distance from $P$ to $Q$. The case when this distance is equal to 0 (the dashed part consists of a unique subarc) will be considered at the end of the proof.

Suppose now that the arc distance from $P$ to $Q$ is at least two. This means that there are several arcs that are dashed or partially dashed. The arc that one encounters first when traveling in the net from $P$ and crossing 
the line at infinity once is below all other arcs. It may have 1-gons if there is a crossing with both branches in this lowest arc, or not.

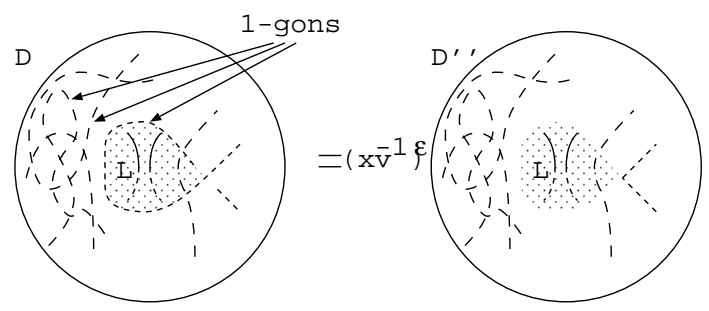

Fig. 18

CASE 1: There are 1-gons in the lowest arc (see Figure 18). Consider $D^{\prime \prime}$ obtained from $D$ by eliminating some 1-gon in the lowest arc. Let $\varepsilon$ be the sign at the self-crossing of this 1-gon. Notice that $H\left(D^{\prime \prime}\right)=\left(x v^{-1}\right)^{w-\varepsilon} H\left(D^{\prime}\right)$ by induction on $k$ ( $D^{\prime \prime}$ has strictly fewer crossings than $\left.D\right)$.

To see that $H(D)=\left(x v^{-1}\right)^{\varepsilon} H\left(D^{\prime \prime}\right)$, one considers the part $L$ of $D$ that is inside the 1-gon (and the corresponding part of $D^{\prime \prime}$ ). In $L$, there may be subarcs below the 1-gon (they can only be parts of the lowest arc). All other subarcs are above the 1-gon, and they are above the subarcs that are below the 1-gon. Lemma 14 can be applied, so $H(D)=\left(x v^{-1}\right)^{\varepsilon} H\left(D^{\prime \prime}\right)$. Thus $H(D)=\left(x v^{-1}\right)^{w} H\left(D^{\prime}\right)$.

CASE 2: There are no 1-gons in the lowest arc. The lowest arc divides $D$ in two parts, with $P$ and $Q$ in one of them. Consider the other part, the good part.

There may be 1-gons that are entirely inside the good part. If some of them are dashed, one gets $H(D)=\left(x v^{-1}\right)^{w} H\left(D^{\prime}\right)$ using Lemma 14 as in Case 1 (here it is essential that $P$ and $Q$ are not inside such 1-gons).

If inside the good part there are only 1-gons that are not dashed, then, using for $D$ and $D^{\prime}$ relation (HI) for crossings that have both branches not in the dashed part, one gets again to a situation where an application of Lemma 14 is possible, whereas for the smoothings that appear in (HI), one uses induction on $k$.

Suppose now that there are no 1-gons in the good part of the lowest arc. Consider a most nested dashed arc inside this part. Two situations can occur: the most nested arc has a good part which either contains a couple of antipodal points on the boundary circle of the diagram (more difficult case), or does not contain such a couple (simpler case). These two cases are shown in Figure 19.

In the simpler case $H(D)$ and $H\left(D^{\prime}\right)$ are computed using relation (HI), by ordering one above the other the non-dashed subarcs inside the good part of the most nested dashed arc. Again, for smoothings one has equality for 


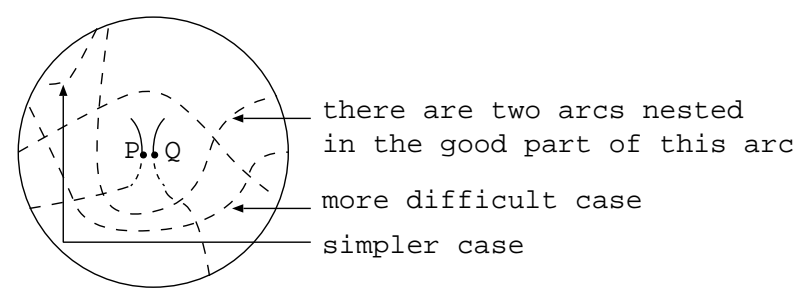

Fig. 19

$H$ (up to $\left(x v^{-1}\right)^{w}$ ) by induction on $k$. For the diagrams where the subarcs are ordered, one gets equality for $H$ (up to $\left(x v^{-1}\right)^{w}$ ) using either Lemma 10 and induction on $k$, or Lemma $9, \Omega_{5}$ and $\Omega_{4}$ moves to make the dashed arc disappear and induction on the arc distance from $P$ to $Q$.

In the more difficult case the situation is presented in Figure 20.

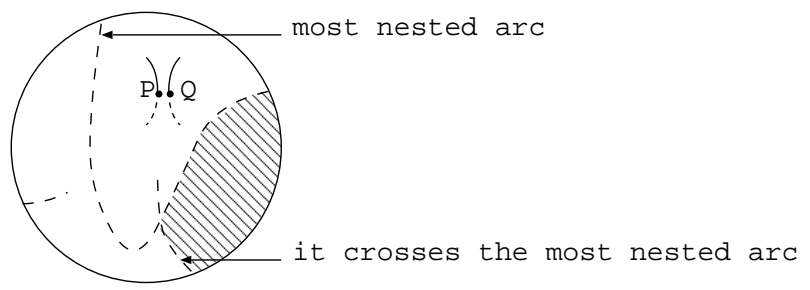

Fig. 20

Consider an arc which has an endpoint antipodal to an endpoint of the most nested dashed arc. Such an arc has to intersect this most nested arc: otherwise it would be nested in it.

Consider the dashed triangle in Figure 20. Order all subarcs inside the triangle with the (HI) relation (for the smoothings use induction on $k$ to get equality for $H$ up to $\left.\left(x v^{-1}\right)^{w}\right)$. If there are 2-gons inside the triangle (including 2-gons formed by a part of a side of the triangle and another subarc), eliminate them without changing $H$ (Lemma 10) and decrease the number of crossings. Then use induction on $k$.

Now, suppose that there are no 2-gons inside the triangle. First, eliminate all subarcs inside the triangle with both endpoints in the boundary circle of the diagram (i.e. all arcs inside the triangle) using Lemma 9 and $\Omega_{5}$ and $\Omega_{4}$ moves.

If the side of the triangle which is part of the boundary circle of the diagram contains no endpoints of arcs except the two vertices of the triangle, then, by Lemma 15, the triangle can be removed without changing $H$ and one gets to the simpler case. 
If the side of the triangle which is part of the boundary circle of the diagram contains endpoints of arcs that are not vertices of the triangle, consider a smaller triangle inside the original one, which has one side in the boundary circle of the diagram and for which the assumptions of Lemma 15 are satisfied. It is easily seen that such a triangle can always be found. The smaller triangle is eliminated without changing $H$. After the elimination the number of crossings inside the original triangle or the number of endpoints of arcs in the original triangle decreases. Repeating this procedure several times, one gets to a situation where there are no endpoints of arcs in the original triangle. Using Lemma 15, one then gets to the simpler case.

InDUCTION BASIS: Suppose that the arc distance from $P$ to $Q$ is equal to zero. This means that there is only one arc partially dashed from $P$ to $Q$.

If there is a crossing with both branches dashed, consider the first such crossing, say $X$, encountered when traveling from $P$ according to the orientation. Let $a$ be the part of $D$ that is covered while traveling in the net from the upper branch to the lower branch of $X$. Let $D^{\prime \prime}$ be obtained from $D$ by erasing $a$. Let $w^{\prime}$ be the sum of the signs of the crossings at which both branches belong to a (including $X$ ). Then, as $D$ is descending from the upper to the lower branch of $X, H(D)=\left(x v^{-1}\right)^{w^{\prime}} H\left(D^{\prime \prime}\right)$ by Lemma 2 . And, by induction on $k, H\left(D^{\prime \prime}\right)=\left(x v^{-1}\right)^{w-w^{\prime}} H\left(D^{\prime}\right)$.

Now, if there are no crossings with both branches dashed, the situation is as in one of the two cases shown in Figure 21.
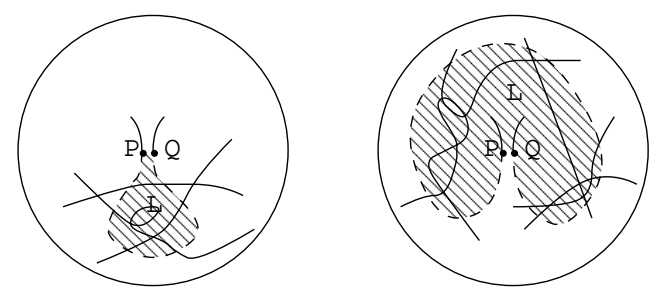

Fig. 21

In these cases the dashed part is above everything else. Again, it is possible to order all non-dashed subarcs inside the dashed part $L$. If there are 1-gons inside $L$ they are eliminated as before. Otherwise, subarcs are removed from $L$ by using Lemma 10 . One gets finally to a situation where there are no non-dashed subarcs inside $L$, except the two subarcs containing $P$ and $Q$ in the second case of Figure 21. Then one checks easily that $H(D)=$ $H\left(D^{\prime}\right)$.

Proposition 6 (invariance under $\Omega_{2}^{\prime}$ moves). $H$ does not change under $\Omega_{2}^{\prime}$ moves involving diagrams with $n$ and $n-2$ crossings. 
Proof. By Remark 2 and Lemma 5, we may suppose that the based diagram involved in an $\Omega_{2}^{\prime}$ move is descending (except possibly at the crossing involved in the move that is not the basepoint). In the following figures, $w$ stands for the sum of the signs of some self-crossings that are removed.

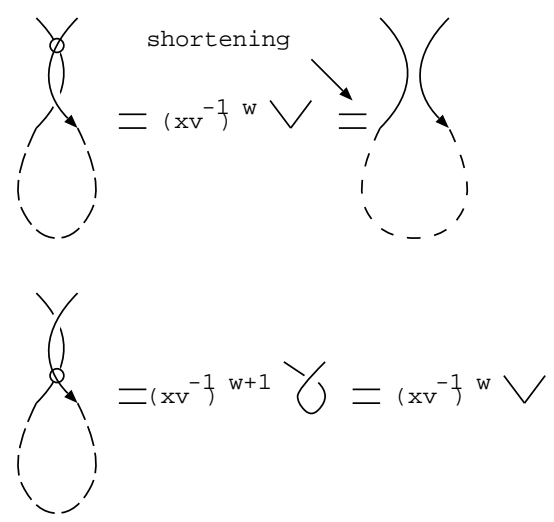

Fig. 22
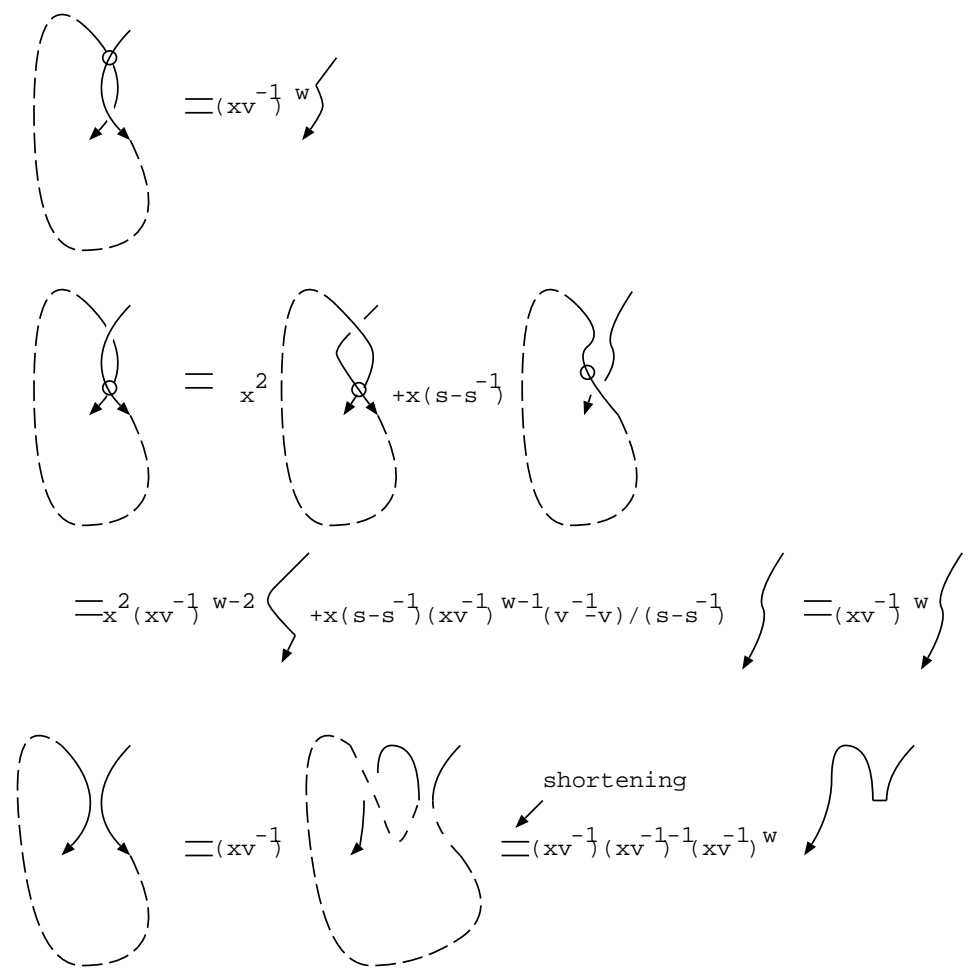

Fig. 23 
There are two cases to consider. In the first case one branch is oriented downwards and the other is oriented upwards. Then $H$ is unchanged as shown in Figure 22.

In the second case both branches involved in the move are oriented downwards, and $H$ is unchanged as shown in Figure 23.

Proposition 7 (invariance under $\Omega_{3}^{\prime}$ moves). $H$ does not change under $\Omega_{3}^{\prime}$ moves involving diagrams with $n$ crossings.
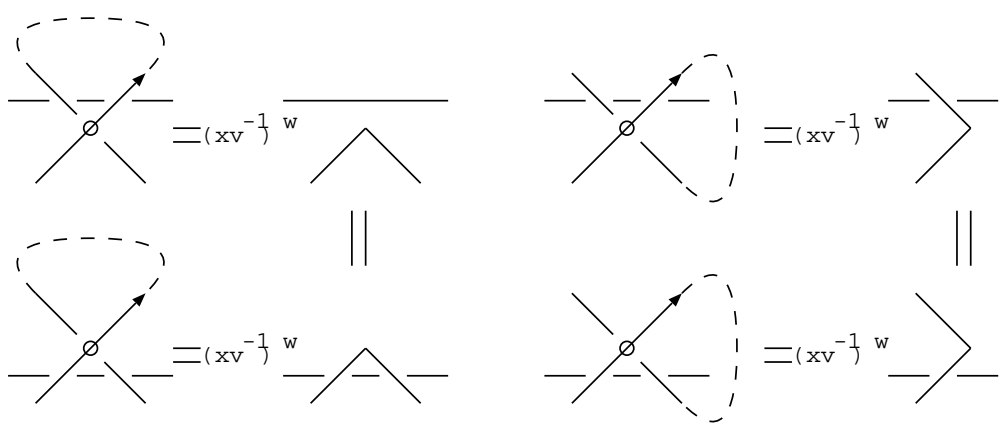

Fig. 24
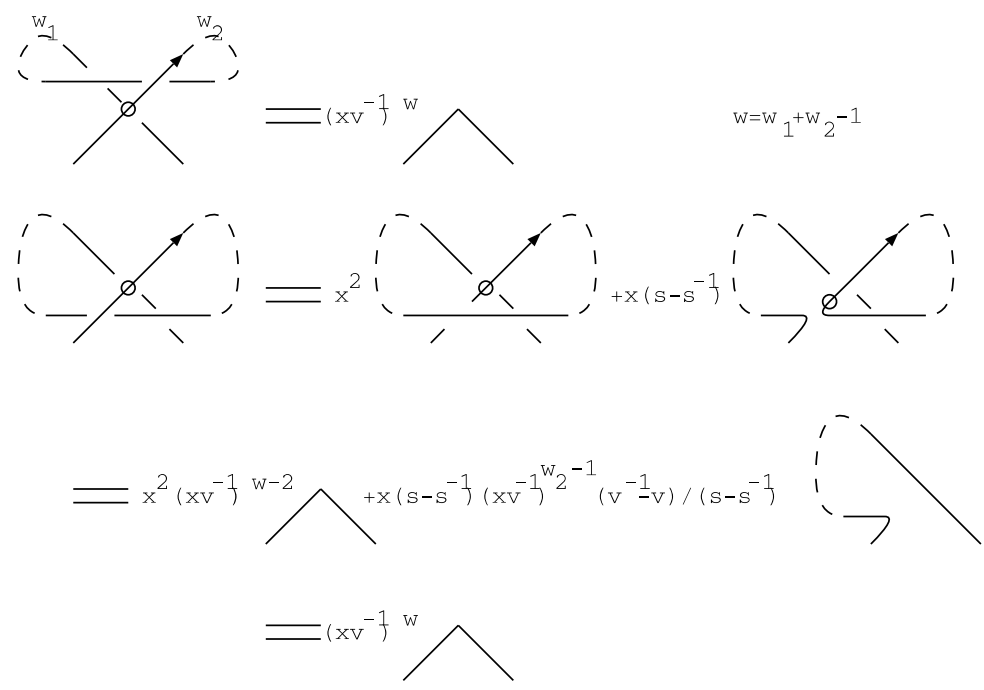

because, by shortening :

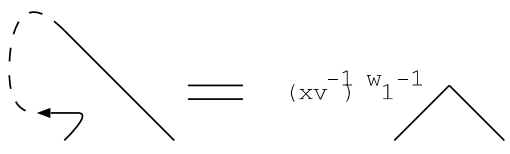

Fig. 25 


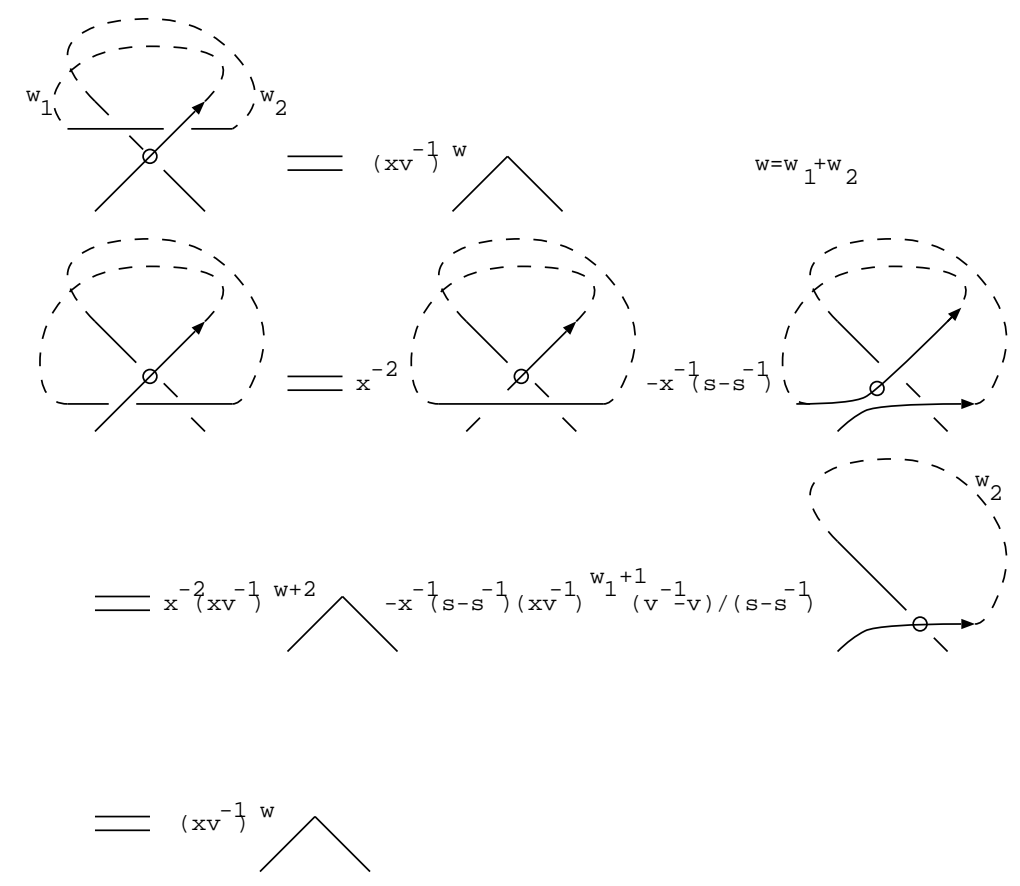

Fig. 26

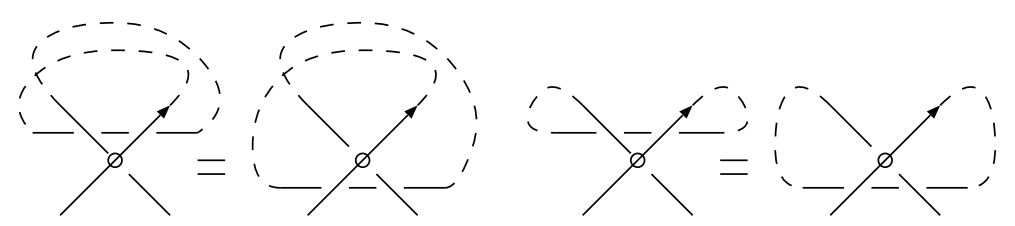

Fig. 27

Proof. By Remark 2, one may suppose that the diagrams involved in the move are descending except possibly at the crossings appearing in the move. Furthermore, using Lemma 5, one may assume that the diagram before the move is descending.

In Figures 24-27, the calculations for $H$ under $\Omega_{3}^{\prime}$ moves are shown. One also has to calculate $H$ under $\Omega_{3}^{\prime}$ moves obtained from the moves presented in these figures by performing reflections with respect to vertical lines passing through the basepoints, but the calculations are similar. This gives all possible $\Omega_{3}^{\prime}$ moves.

In Figures 25 and 26 one supposes that the arc distance from the basepoint to the lowest branch is even, whereas in Figure 27 it is odd; $w, w_{1}$ and $w_{2}$ stand for the sums of the signs of some self-crossings that are removed. 
4.7. $\Omega_{4}^{\prime \prime}$ and $\Omega_{5}^{\prime \prime \prime}$ moves. A diagram $D$ is said to be ascending from $P$ to $Q$ if $D^{\prime}$ is descending from $P$ to $Q$, where $D^{\prime}$ is obtained from $D$ by switching every crossing.

For a non-simple diagram $\dot{D}$, denote by $w(\dot{D})$ the sum of the signs at the self-crossings of the 0-homologous component with basepoint; or the sum of the signs at the self-crossings of the dashed part determined by the basepoint.

Lemma 16 (shortening of some based diagrams). Consider based diagrams $\dot{D}_{1}$ and $\dot{D}_{2}$ with at most $n$ crossings, presented in Figure 28 . Suppose that $\dot{D}_{1}$ is ascending from $P$ to $Q$. Let $D_{1}^{\prime}$ be obtained from $\dot{D}_{1}$ by removing the 0-homologous component with basepoint. Then

$$
H\left(\dot{D}_{1}\right)=\mu\left(x v^{-1}\right)^{w\left(\dot{D}_{1}\right)} H\left(D_{1}^{\prime}\right) .
$$

Suppose that the dashed part determined by the basepoint of $\dot{D}_{2}$ is ascending, i.e. $\dot{D}_{2}$ is ascending from $P$ to $Q$. Let $D_{2}^{\prime}$ be obtained from $\dot{D}_{2}$ by removing the dashed part determined by the basepoint. Then

$$
H\left(\dot{D}_{2}\right)=\left(x v^{-1}\right)^{w\left(\dot{D}_{2}\right)} H\left(D_{2}^{\prime}\right) .
$$
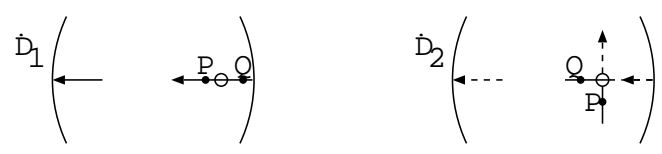

Fig. 28

Proof. The proof is similar to that of Proposition 5. It is by induction on the number of crossings in $\dot{D_{1}}$ and $\dot{D_{2}}$. If this number is 0 in the case of $\dot{D}_{1}$ or 1 in the case of $\dot{D}_{2}$ then the lemma follows from the definition of $H$.

Assuming that the assertion is true for diagrams with fewer than $k$ crossings, it is proven for diagrams with $k$ crossings by induction on $l$, the arc distance from $P$ to $Q$.

It is clear from Figure 28 that $l$ is at least 2 . If there are some 1-gons, one uses (HI), induction on $k$ and Lemma 14 to eliminate them (see the proof of Proposition 5, Case 1). Any 1-gon can be eliminated in this way, because $P$ and $Q$ are not inside any 1-gon. If there are no 1-gons, one reduces the arc distance from $P$ to $Q$ in the same way as in the proof of Proposition 5, Case 2, by eliminating the highest arc (the one encountered when traveling in the net from $P$ and crossing the line at infinity once), while keeping the part of the diagram near $P$ and $Q$ unchanged.

Finally, if $l=2$ and there are no 1-gons, one reduces the arc distance from $P$ to $Q$ to 0 . Then, using Lemma 9, one reduces the number of crossings involving the component with basepoint to 0 in the case of $\dot{D}_{1}$; or one 
reduces the number of crossings involving the dashed part determined by the basepoint to 1 in the case of $\dot{D}_{2}$. The conclusion of the lemma follows from the definition of $H$.

Proposition 8 (invariance under $\Omega_{4}^{\prime \prime}$ and $\Omega_{5}^{\prime \prime \prime}$ moves). $H$ does not change under $\Omega_{4}^{\prime \prime}$ and $\Omega_{5}^{\prime \prime \prime}$ moves involving diagrams with $n$ crossings.

Proof. Consider first an $\Omega_{4}^{\prime \prime}$ move. Notice that it can be obtained with the crossing of the line at infinity in the net by the basepoint followed by a good $\Omega_{4}$ move. It follows from Lemmas 3 and 16 that $H$ does not change when the basepoint crosses the line at infinity in the net (before this crossing the component with basepoint may be assumed descending, and it becomes ascending after the crossing). Also $H$ does not change under good Reidemeister moves. Thus $H$ does not change under an $\Omega_{4}^{\prime \prime}$ move.

Notice that Lemma 16 is also true if in the diagram $\dot{D}_{2}$ presented in Figure 28, the vertical branch of the basepoint points downwards instead of upwards.

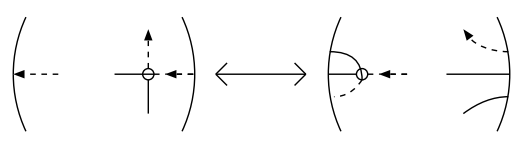

Fig. 29

Consider now an $\Omega_{5}^{\prime \prime \prime}$ move. It can be obtained with a good $\Omega_{4}$ move and the crossing by the basepoint of the line at infinity, presented in Figure 29 (or a similar crossing for which the vertical branch of the basepoint on the left of Figure 29 points downwards instead of upwards). It follows from Lemmas 3 and 16 that $H$ does not change when the basepoint crosses the line at infinity in the net (before this crossing the dashed part determined by the basepoint may be assumed descending, and it becomes ascending after the crossing). Also $H$ does not change under good moves. Thus $H$ does not change under an $\Omega_{5}^{\prime \prime \prime}$ move.

We have established that $H$ does not change under any Reidemeister move that does not increase the number of crossings beyond $n$.

\subsection{Basepoints of non-simple diagrams}

Proposition 9 (independence from basepoints for non-simple diagrams). Let $\dot{D}_{1}$ and $\dot{D}_{2}$ be two non-simple based diagrams with $n$ crossings. Suppose that $\dot{D}_{1}$ and $\dot{D}_{2}$ differ only in the position of the basepoint. Then $H\left(\dot{D}_{1}\right)=$ $H\left(\dot{D}_{2}\right)$.

Proof. First, suppose that there are some 2-gons or 1-gons in $\dot{D}_{1}$. Notice that as $H$ does not change under any Reidemeister move that does not increase the number of crossings beyond $n$, Lemmas $9-11$ can be extended 
to the situation where there are no restrictions on the position of the basepoint. Using Lemma 3 and the extended versions of Lemmas 10 and 11 one may reduce the number of crossings in $\dot{D}_{1}$ and $\dot{D}_{2}$. Thus $H\left(\dot{D}_{1}\right)=H\left(\dot{D}_{2}\right)$ by $\operatorname{IH}(n-1)$.

It can be easily seen that, if there are no 1 -gons and no 2 -gons in $\dot{D}_{1}$, then there has to be an arc with endpoints on the boundary circle of the diagram that are not antipodal. Let $n$ be the number of such arcs in $\dot{D}_{1}$.

The proof is by induction on $n$. If $n=0$ then there are some 2-gons or 1-gons in $\dot{D}_{1}$. If there are $n$ arcs in $\dot{D}_{1}$ and $\dot{D}_{2}$, then one arc can be removed by using Lemma 3 , the extended version of Lemma 9 and $\Omega_{5}$ and $\Omega_{4}$ moves. Thus, by induction on $n, H\left(\dot{D}_{1}\right)=H\left(\dot{D}_{2}\right)$.

This was the final step in the proof of the induction hypothesis $\operatorname{IH}(n)$. Theorem 2 follows.

\section{INDUCTIVE DEFINITION OF THE KAUFFMAN POLYNOMIAL $K$}

The proof of Theorem 4 is similar to that of Theorem 2 above. The differences in the proofs are as in the case of links in $\mathbb{R}^{3}$. The parts of the proofs that are specific to the $\mathbb{R} P^{3}$ situation are almost identical for Homfly and Kauffman polynomials.

$K$ is defined in a similar way to $H$ (see Section 3). The definition is in fact simpler because in the unoriented case there is a good notion of descending diagram (see [9]).

The standard diagram of a standard unoriented unlink $L_{n}$ is the diagram in Figure 1 with the orientations being disregarded. Let $d=\left(a+a^{-1}\right) z^{-1}-1$.

5.1. Inductive hypothesis $\operatorname{IH}(n-1)$. There is a function $K$ defined on the set of diagrams with at most $n-1$ crossings, taking values in $\mathbb{Z}\left[a^{ \pm 1}, z^{ \pm 1}, y\right]$, such that:

(1) $K$ is invariant under those Reidemeister moves that do not increase the number of crossings beyond $n-1$.

(2) $K$ satisfies relations (KI) and (KII).

(3) If $D$ is the standard diagram of the standard unoriented unlink $L_{m}$, $m>0$, with at most $n-1$ crossings (i.e. $m(m-1) / 2 \leq n-1)$, then $K(D)=y^{m}$. Also, $K(\bigcirc)=d$.

5.2. Diagrams with no crossings. Let $D$ be a diagram with 0 crossings. Let $p$ be the number of its 0 -homologous components and $m$ the number of its 1-homologous components ( $m$ is 0 or 1$)$. Then, by definition,

$$
K(D)=d^{p} y^{m} \text {. }
$$

Note that $K$ satisfies $\mathrm{IH}(0)$. 
5.3. Diagrams with $n \geq 1$ crossings. We assume that the inductive hypothesis $\mathrm{IH}(n-1)$ holds true.

As in the case of the polynomial $H$, the construction of $K$ for diagrams with $n$ crossings is divided into several cases treated in the subsequent subsections. In each case a diagram $D$ with $n$ crossings is endowed with some extra structure (a directed basepoint or a couple of basepoints). $D$ together with this structure is denoted by $\dot{D}$. A diagram $\alpha(\dot{D})$ is then defined; it is obtained from $\dot{D}$ by a series of crossing changes.

The diagram $\alpha(\dot{D})$ has the following property: if $X$ is one of the crossings of $\dot{D}$ that have to be switched to obtain $\alpha(\dot{D})$, and $\dot{D}^{\prime}$ is obtained from $\dot{D}$ by switching $X$, then $\alpha(\dot{D})=\alpha\left(\dot{D}^{\prime}\right)$.

In the following subsections $K$ is defined on $\alpha(\dot{D})$ for each case (see $\left(K_{3}\right)$ to $\left.\left(K_{5}\right)\right)$.

Suppose that $K$ is already defined on all $\alpha(\dot{D})$. For a based diagram $\dot{D}$, denote by $S(\dot{D})$ the set of crossings of $\dot{D}$ where $\dot{D}$ and $\alpha(\dot{D})$ differ. Let $k$ be the number of elements in $S(\dot{D})$ and $\omega$ a (linear) ordering of $S(\dot{D})$. Denote by $S(\dot{D}, \omega)$ the set $S(\dot{D})$ equipped with the ordering $\omega$.

We define $K(\dot{D}, \omega)$ by induction on $k$. The definition depends on $\omega$. If $k=0$ then $K$ is already defined. Otherwise let $\dot{D}^{\prime}$ be the based diagram obtained from $\dot{D}$ by switching the first crossing in $S(\dot{D}, \omega)$; let $D_{1}^{\prime \prime}$ and $D_{2}^{\prime \prime}$ be the diagrams obtained by smoothing the same crossing in two possible ways. Let $\omega^{\prime}$ be the ordering of all crossings of $S\left(\dot{D}^{\prime}\right)$ induced by $\omega$. Note that there are $k-1$ elements in $S\left(\dot{D}^{\prime}\right) . K\left(\dot{D}^{\prime}, \omega^{\prime}\right)$ is defined by induction on $k$, and $K\left(D_{1}^{\prime \prime}\right)$ and $K\left(D_{2}^{\prime \prime}\right)$ are defined by $\operatorname{IH}(n-1)$. Now $K(\dot{D}, \omega)$ is defined using relation (KI) for the first crossing in $S(\dot{D}, \omega)$ with the help of $K\left(\dot{D}^{\prime}, \omega^{\prime}\right), K\left(D_{1}^{\prime \prime}\right)$ and $K\left(D_{2}^{\prime \prime}\right)$. By definition,

$$
K(\dot{D}, \omega)=-K\left(\dot{D}^{\prime}, \omega^{\prime}\right)+z\left(K\left(D_{1}^{\prime \prime}\right)+K\left(D_{2}^{\prime \prime}\right)\right) .
$$

5.4. Simple diagrams. The definition of a simple diagram is the same in the oriented and unoriented cases (see Subsection 3.4). The same is true for the definitions of based simple diagram and primary and secondary basepoints.

In the case of unoriented links, a based simple diagram $\dot{D}$ is said to be descending if, whenever it is oriented in any way, it is descending (see end of Section 3.4). It can be easily seen that this definition does not depend on the orientation. For a based simple diagram $\dot{D}$, let $\alpha(\dot{D})$ be the based diagram obtained from $\dot{D}$ by the crossing changes that make it descending.

Let $p$ be the number of 0 -homologous components and $m$ the number of 1-homologous components of $\dot{D}$. By definition,

$$
K(\alpha(\dot{D}))=d^{p} y^{m} .
$$


5.5. Non-simple diagrams. In the case of non-simple diagrams, a basepoint for an unoriented link is defined as a basepoint for an oriented link (see Section 3.5); moreover, it is endowed with an arrow giving a local orientation at the basepoint. In the case of a basepoint which is a selfcrossing of a 1-homologous component the local orientation is given to one of its branches. A basepoint together with an arrow is called a directed basepoint.

The notion of a non-simple descending diagram in the oriented case depends only on the orientation of the component with basepoint (see Section 3.5). In the unoriented case, a non-simple based diagram $\dot{D}$ is descending if, whenever we endow the component on which the directed basepoint lies with the orientation given by the arrow of this basepoint, it is descending in the oriented sense. The diagram $\alpha(\dot{D})$ is the based diagram obtained from $\dot{D}$ by the crossing changes which are necessary to make it descending.

Let $\dot{D}$ be a based diagram for which the directed basepoint is on a 0-homologous component. Let $D^{\prime}$ be obtained from $\dot{D}$ by removing this component (if $D^{\prime}$ is empty then $K\left(D^{\prime}\right)$ is replaced by 1 in $\left(K_{4}\right)$ below). Let $w$ be the sum of all signs at all self-crossings of the component with basepoint in $\alpha(\dot{D})$, where this component is oriented arbitrarily. Then, by definition,

$$
K(\alpha(\dot{D}))=d a^{w} K\left(D^{\prime}\right) .
$$

Let $\dot{D}$ be a based diagram for which the directed basepoint is a selfcrossing of a 1-homologous component. Let $D^{\prime}$ be obtained from $\dot{D}$ by removing the dashed part determined by the basepoint. Endowing the component with basepoint with an arbitrary orientation, let $w$ be the sum of all signs at all self-crossings of the dashed part determined by the basepoint of $\alpha(\dot{D})$, including the basepoint (which is a self-crossing). Then, by definition,

$$
K(\alpha(\dot{D}))=a^{w} K\left(D^{\prime}\right) .
$$

\section{INDEPENDENCE FROM CHOICES FOR $K$, INVARIANCE UNDER REIDEMEISTER MOVES}

\subsection{Relations (KI) and (KII)}

LEMMA 17 (independence from ordering). Let $\dot{D}$ be a based diagram with $n$ crossings. Let $\omega$ and $\omega^{\prime}$ be two orderings of the set of crossings of $\dot{D}$ that are different in $\dot{D}$ and $\alpha(\dot{D})$. Then $K(\dot{D}, \omega)=K\left(\dot{D}, \omega^{\prime}\right)$.

Proof. By induction on the number of crossing differences between $\dot{D}$ and $\alpha(\dot{D})$ it is sufficient to prove that $K$ does not change if one switches the first two crossings according to $\omega$, say $C_{1}$ and $C_{2}$. 
Denote by $\sigma_{i} \dot{D}$ the diagram obtained from $\dot{D}$ by switching $C_{i}(i=1,2)$. Denote by $\mu_{i} \dot{D}$ and $\nu_{i} \dot{D}$ the diagrams obtained from $\dot{D}$ by smoothing $C_{i}$ $(i=1,2)$ in two different ways. Here it does not matter which smoothing is $\mu_{i} \dot{D}$ and which is $\nu_{i} \dot{D}$. First consider the sequence in which $C_{1}$ is switched before $C_{2}$ :

$$
\begin{aligned}
K(\dot{D}, \omega) & =-K\left(\sigma_{1} \dot{D}\right)+z K\left(\mu_{1} \dot{D}\right)+z K\left(\nu_{1} \dot{D}\right) \\
& =K\left(\sigma_{2} \sigma_{1} \dot{D}\right)-z K\left(\mu_{2} \sigma_{1} \dot{D}\right)-z K\left(\nu_{2} \sigma_{1} \dot{D}\right)+z K\left(\mu_{1} \dot{D}\right)+z K\left(\nu_{1} \dot{D}\right) .
\end{aligned}
$$

Switching $C_{2}$ before $C_{1}$ yields

$$
\begin{aligned}
K\left(\dot{D}, \omega^{\prime}\right) & =-K\left(\sigma_{2} \dot{D}\right)+z K\left(\mu_{2} \dot{D}\right)+z K\left(\nu_{2} \dot{D}\right) \\
& =K\left(\sigma_{1} \sigma_{2} \dot{D}\right)-z K\left(\mu_{1} \sigma_{2} \dot{D}\right)-z K\left(\nu_{1} \sigma_{2} \dot{D}\right)+z K\left(\mu_{2} \dot{D}\right)+z K\left(\nu_{2} \dot{D}\right) .
\end{aligned}
$$

Now,

$$
\begin{aligned}
K(\dot{D}, \omega)-K\left(\dot{D}, \omega^{\prime}\right)= & z\left(-K\left(\mu_{2} \sigma_{1} \dot{D}\right)-K\left(\mu_{2} \dot{D}\right)-K\left(\nu_{2} \sigma_{1} \dot{D}\right)-K\left(\nu_{2} \dot{D}\right)\right. \\
& \left.+K\left(\mu_{1} \dot{D}\right)+K\left(\mu_{1} \sigma_{2} \dot{D}\right)+K\left(\nu_{1} \dot{D}\right)+K\left(\nu_{1} \sigma_{2} \dot{D}\right)\right)
\end{aligned}
$$

By $\operatorname{IH}(n-1)$, one can use (KI) for diagrams with $n-1$ crossings, so

$$
\begin{aligned}
& K(\dot{D}, \omega)-K\left(\dot{D}, \omega^{\prime}\right) \\
& \quad=z^{2}\left(-K\left(\mu_{2} \mu_{1} \dot{D}\right)-K\left(\mu_{2} \nu_{1} \dot{D}\right)-K\left(\nu_{2} \mu_{1} \dot{D}\right)-K\left(\nu_{2} \nu_{1} \dot{D}\right)\right. \\
& \left.\quad+K\left(\mu_{1} \mu_{2} \dot{D}\right)+K\left(\mu_{1} \nu_{2} \dot{D}\right)+K\left(\nu_{1} \mu_{2} \dot{D}\right)+K\left(\nu_{1} \nu_{2} \dot{D}\right)\right)=0 .
\end{aligned}
$$

Lemma 2 can be easily modified to the case of $K$ :

Lemma 18. Let $D$ be a diagram with at most $n-1$ crossings and let $X$ be a self-crossing of a component $b$ of $D$. Suppose that for some fixed orientation of $b$, the arc distance from the upper to the lower branch of $X$ is even and $D$ is descending from the upper to the lower branch of $X$. Let $b^{\prime}$ be the part of $b$ that is covered if one travels in the net from the upper to the lower branch of $X$, according to the fixed orientation of $b$. Let $D^{\prime}$ be obtained from $D$ by erasing $b^{\prime}$. Let $w$ be the sum of the signs of the self-crossings of $b^{\prime}$ (including $\left.X\right)$. Then $K(D)=a^{w} K\left(D^{\prime}\right)$.

In the same way as for $H$, from the preceding lemma and Lemma 17 follows:

Proposition 10 (Kauffman relations). Relation (KII) holds for $K$ whenever the diagram on the left has $n$ crossings. Relation (KI) holds for $K$ whenever the two based diagrams on the left have $n$ crossings and have the same basepoint $(s)$.

6.2. Basepoints for $\mathbf{0}$-homologous components. Proposition 5 for $H$ is a consequence of the inductive hypothesis $\operatorname{IH}(n-1)$ only. It can be easily modified to the case of $K$ : 
Proposition 11 (shortening of diagrams). Let $D$ be a diagram with $k$ crossings. A part of $D$ is shown on the left of Figure 30. Suppose that, if the component of $D$ which contains $P$ and $Q$ is oriented in such a way that one can travel in the net from $P$ to $Q$ while covering the dashed part on the left of Figure 30, then the arc distance from $P$ to $Q$ is even and $D$ is descending from $P$ to $Q$. Let $D^{\prime}$ be obtained from $D$ by removing the dashed part and joining $P$ and $Q$ with a segment ( $a$ part of $D^{\prime}$ is shown on the right of Figure 30). Let $w$ be the sum of the signs at all crossings for which both branches are in the dashed part. If $k \leq n-1$ then $K(D)=a^{w} K\left(D^{\prime}\right)$.

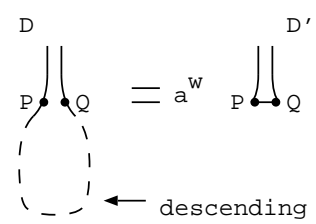

Fig. 30

Lemma 19 (moving the basepoint). Suppose that $P$ is a directed basepoint lying on a 0-homologous component $b$ of a based diagram $D$ with $n$ crossings and $P$ is on an arc $c$. Then $K$ does not change if $P$ moves on $c$.

Proof. It is sufficient to prove that $K$ is unchanged if the basepoint passes through a crossing as in Figure 31.
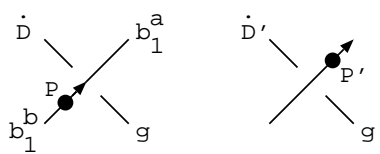

Fig. 31

Notice that, as relation (KI) holds for diagrams with $n$ crossings, if $K$ does not change for some $D$ when moving the basepoint, then it does not change for any diagram obtained from $D$ by some crossing changes (by $\mathrm{IH}(n-1))$. We may therefore suppose that the based diagram $\dot{D}$ on the left of Figure 31 is descending. Starting from $P$ and traveling on the net of $D$ according to the orientation given by the arrow of the basepoint, denote the successive arcs encountered by $b_{1}, \ldots, b_{l}$. Furthermore denote the part of $b_{1}$ that comes after $P$ by $b_{1}^{a}$ and the remaining part by $b_{1}^{b}$. Just as in the proof of Lemma 4 one has

$$
b_{2} \leq b_{4} \leq b_{6} \leq \cdots \leq b_{1}^{b} \leq \cdots \leq b_{5} \leq b_{3} \leq b_{1}^{a},
$$

where $b_{i} \leq b_{j}$ means that $b_{i}$ is below $b_{j}$. 
Now $\dot{D}^{\prime}$ may be descending or not. If it is descending then $K(\dot{D})=$ $K\left(\dot{D}^{\prime}\right)$. It is not descending if and only if the branch $g$ (see Figure 31 ) is a part of $b_{1}^{a}, b_{1}^{b}$ or $b_{k}$ with $k$ odd.

Suppose that $\dot{D}^{\prime}$ is not descending. Notice that it becomes descending if one switches the crossing in Figure 31. Let $\sigma \dot{D}^{\prime}, \eta \dot{D}^{\prime}$ and $\eta_{2} \dot{D}^{\prime}$ be the diagrams obtained from $\dot{D}^{\prime}$ by doing respectively the switching at this crossing, the smoothing at this crossing respecting any orientation of $b$, and the other possible smoothing at the crossing. Note that in $\eta \dot{D}^{\prime}, b$ becomes a link with two 0-homologous components $b^{1}$ and $b^{2}$, where $b^{1}$ contains $P^{\prime}$ and $b^{2}$ contains $P\left(P\right.$ and $P^{\prime}$ can naturally be viewed in $\left.\eta \dot{D}^{\prime}\right)$. Notice that $\eta \dot{D}^{\prime}$ is descending with respect to $P^{\prime}$.

Denote by $D_{b}$ the diagram obtained from $\dot{D}^{\prime}$ by removing $b$, which is the same as the diagram obtained from $\eta \dot{D}^{\prime}$ by removing $b^{1}$ and $b^{2}$. Let $\varepsilon$ be the sign of the crossing in Figure 31 and $w$ the sum of the signs of the crossings for which both branches are in $b$.

Denote by $D_{b^{1}}$ the diagram obtained from $\eta \dot{D}^{\prime}$ by removing $b^{1}$. As $D_{b^{1}}$ is descending with respect to $P$, one has $K\left(\eta \dot{D}^{\prime}\right)=d^{2} a^{w-\varepsilon} K\left(D_{b}\right)$.

In $\eta_{2} \dot{D}^{\prime}$, there is a single 0 -homologous component coming from $b$. It is not descending, as part of it is descending and the other part is ascending. One uses Proposition $11\left(\eta_{2} \dot{D}^{\prime}\right.$ has $n-1$ crossings $)$ to eliminate the descending part. $K$ of the remaining ascending part can be computed by changing the direction of the directed basepoint $P$ so that this part becomes descending (by $\operatorname{IH}(n-1), K$ does not depend on the choice of the directed basepoint). One gets $K\left(\eta_{2} \dot{D}^{\prime}\right)=d a^{w-\varepsilon} K\left(D_{b}\right)$.

Then

$$
\begin{aligned}
K(\dot{D}) & =d a^{w} H\left(D_{b}\right) \\
K\left(\dot{D}^{\prime}\right) & =-K\left(\sigma \dot{D}^{\prime}\right)+z\left(K\left(\eta \dot{D}^{\prime}\right)+K\left(\eta_{2} \dot{D}^{\prime}\right)\right) \\
& =-d a^{w-2 \varepsilon} K\left(D_{b}\right)+z\left(d^{2} a^{w-\varepsilon} K\left(D_{b}\right)+d a^{w-\varepsilon} K\left(D_{b}\right)\right) \\
& =-d a^{w-2 \varepsilon} K\left(D_{b}\right)+z d\left(d a^{w-\varepsilon}+a^{w-\varepsilon}\right) K\left(D_{b}\right) \\
& =d\left(-a^{w-2 \varepsilon}+z\left(a^{w-\varepsilon}\left(a+a^{-1}\right) z^{-1}-a^{w-\varepsilon}+a^{w-\varepsilon}\right)\right) K\left(D_{b}\right) \\
& =d a^{w} K\left(D_{b}\right)
\end{aligned}
$$

as $d=\left(a+a^{-1}\right) z^{-1}-1$.

Thus $K(\dot{D})=K\left(\dot{D}^{\prime}\right)$.

\subsection{Invariance of $K$ under good and bad Reidemeister moves.} Independence from basepoints. The invariance of $K$ under good Reidemeister moves is proved similarly to the invariance of $H$. The calculations that have to be done are the same as in the case of the Kauffman polynomial for classical links [7]. From this it follows, as in the case of $H$, that 

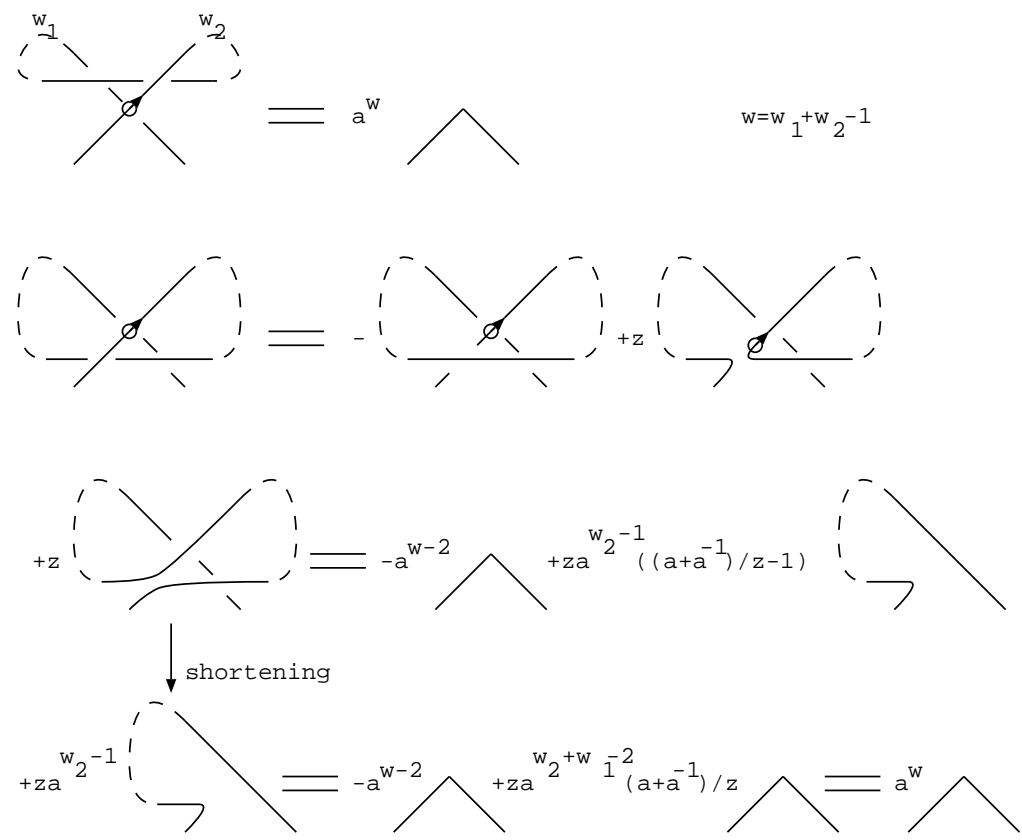

Fig. 32

$K$ is independent of basepoints for simple diagrams (this is Proposition 3 modified to $K$ ).

$K$ is also unchanged under bad Reidemeister moves. An example of calculation is shown in Figure 32. In this figure the arc distance from the basepoint to the middle branch involved in the move is even.

For $K$, the independence from directed basepoints for non-simple diagrams is proven exactly as for $H$ in Proposition 9.

We have established:

Proposition 12 (invariance under Reidemeister moves, independence from basepoints). $K$ does not change under any Reidemeister move that involves diagrams with at most $n$ crossings. For diagrams with $n$ crossings, $K$ does not depend on basepoints.

Thus, assuming that $K$ satisfies $\operatorname{IH}(n-1)$, we have shown that it satisfies $\mathrm{IH}(n)$. Theorem 4 follows.

\section{AN APPLICATION: DISTANCE FROM AFFINITY}

The distance from affinity of a link in $\mathbb{R} P^{3}$ is, by definition, the minimum over all its diagrams of the number of times the line at infinity is intersected in the net. For example, a link is affine if and only if its distance from affinity is equal to 0 . 
The Homfly and Kauffman polynomials can be used to get a lower bound for the distance from affinity of a link:

Proposition 13. Let $L$ be a framed oriented link and suppose that $H(L)$ has degree $n$ in $z$. Then the distance from affinity of $L$ is at least $n$. Let $L$ be a framed unoriented link and suppose that $K(L)$ has degree $n$ in $y$. Then the distance from affinity of $L$ is at least $n$.

Proof. The proof is the same for $H$ and $K$. Suppose that $L$ is a framed oriented link and that $H(L)$ has degree $n$ in $z$. Suppose that there is a diagram of $L$ in which the line at infinity is intersected in the net less than $n$ times. Then, computing $H$ of this diagram does not give rise to terms with degree in $z$ greater than or equal to $n$, by definition of $H$. But in that case $H(L)$ cannot have degree $n$ in $z$.

The proposition above can be used to show that, for any $n \in \mathbb{N} \cup\{0\}$, there exist knots with distance from affinity equal to $n$. An example for $n=5$ is shown in Figure 33. By definition, the distance from affinity of this knot is at most 5 . To see that it is at least 5 , use the Homfly skein relation (HI) successively on the 4 crossings marked with a point in this figure, and get $H\left(L_{5}\right)$ with a factor $x^{4}\left(s-s^{-1}\right)^{4}$ from the smoothings. For the links coming from crossing changes that appear when using (HI), it can be easily seen that their distance from affinity is at most $3 \mathrm{so}$, in $H$, they do not contribute to the term of degree 5 in $z$.

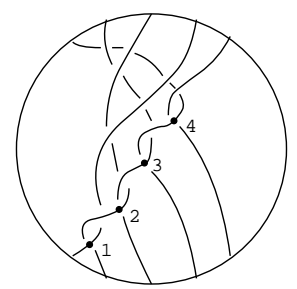

Fig. 33. A knot with distance from affinity equal to 5

An interesting question is whether $H$ or $K$ can detect exactly the distance from affinity of any link.

\section{References}

[1] V. Chernov, Framed knots in 3-manifolds and affine self-linking numbers, preprint arXiv:math.GT/0105139.

[2] Yu. V. Drobotukhina, An analogue of the Jones polynomial for links in $\mathbb{R} P^{3}$ and a generalization of the Kauffman-Murasugi theorem, Algebra i Analiz 2 (1990), no. 3, 171-191 (in Russian); English transl.: Leningrad Math. J. 2 (1991), 613-630. 
[3] P. Freyd, D. Yetter, J. Hoste, W. B. R. Lickorish, K. Millett and A. Ocneanu, A new polynomial invariant of knots and links, Bull. Amer. Math. Soc. 12 (1985), 239-246.

[4] J. Hoste and J. Przytycki, Homotopy skein modules of orientable 3-manifolds, Math. Proc. Cambridge Philos. Soc. 108 (1990), 475-488.

[5] —, - , The $(2, \infty)$-skein module of lens spaces; a generalization of the Jones polynomial, J. Knot Theory Ramifications 2 (1993), 321-333.

[6] V. F. R. Jones, A polynomial invariant for knots via von Neumann algebras, Bull. Amer. Math. Soc. 12 (1985), 103-111.

[7] L. H. Kauffman, An invariant of regular isotopy, Trans. Amer. Math. Soc. 318 (1990), 417-471.

[8] W. B. R. Lickorish and K. C. Millett, A polynomial invariant of oriented links, Topology 26 (1987), 107-141.

[9] M. Mroczkowski, Diagrammatic unknotting of knots and links in the projective space, J. Knot Theory Ramifications 12 (2003), 637-651.

[10] J. H. Przytycki, Skein modules of 3-manifolds, Bull. Polish Acad. Sci. Math. 39 (1991), 91-100.

[11] V. G. Turaev, The Conway and Kauffman modules of the solid torus, Zap. Nauchn. Sem. Leningrad. Otdel. Mat. Inst. Steklov. (LOMI) 167 (1988), 79-89 (in Russian); English tansl.: J. Soviet Math. 52 (1990), 2799-2805.

Department of Mathematics

Uppsala University

75106 Uppsala, Sweden

E-mail: mroczkow@math.uu.se

Received 10 April 2004;

in revised form 27 August 2004 